\title{
Religion und gesellschaftliche Differenzierung: Sozialhistorische Analysen zur Emergenz der europäischen Moderne
}

\author{
Detlef Pollack
}

Online publiziert: 30. April 2020

(C) Der/die Autor(en) 2020

Zusammenfassung Das Ziel des vorliegenden Aufsatzes besteht darin zu prüfen, inwieweit die soziologische Differenzierungstheorie das Potential besitzt, den Beitrag der Religion zur Herausbildung der europäischen Moderne verständlich zu machen. Um die Frage nach dem religiösen Einfluss auf die Entstehung der modernen Welt differenzierungstheoretisch behandeln zu können, ist es zunächst erforderlich, das gebrauchte theoretische Instrumentarium zu erläutern. Es muss dargelegt werden, worin die wesentlichen Aussagen der Differenzierungstheorie bestehen, und es ist notwendig anzugeben, welcher Begriff von Moderne vorausgesetzt wird. Daraufhin erfolgt die historische Analyse.

Methodologisch stellt der Text insofern eine Verbindung zwischen problemorientierten theoretischen Grundsatzüberlegungen und historisch-hermeneutischen Interpretationen dar.

Als Resultat der Untersuchung können wir feststellen, dass sich am Ende des 18. Jahrhunderts die Umrisse einer sich neu herausbildenden Gesellschaftsformation erkennen lassen: einer funktional differenzierten Gesellschaft. Sowohl auf der sozialstrukturellen Ebene als auch auf der Ebene des Diskurses sind zu diesem Zeitpunkt die Prozesse einer Differenzierung unterschiedlicher Wert- und Gesellschaftssphären weit vorangeschritten. Ging die römische Kirche im Hochmittelalter in der Behauptung einer sozial unableitbaren theologischen Eigenrationalität der Entstehung von Differenzierungsformen voran, so bildeten sich in jahrhundertelangen gegenläufigen Prozessen zum Ausgang des 18. Jahrhunderts die Weichen für die Emergenz der europäischen Moderne heraus.

Schlüsselwörter Funktionale Differenzierung · Religion · Emergenz der Moderne $\cdot$ Konflikt $\cdot$ Europäische Moderne 


\title{
Religion and social differentiation: Socio-historical analyses of the emergence of European modernity
}

\begin{abstract}
This article aims at testing the analytical potential the sociological theory of differentiation offers in understanding the contribution of religion for the emergence of European modernity. In order to be able to deal with this question, it is as a start necessary to explicate the theoretical means to be used. It has to be explained firstly what are the components of the sociological theory of differentiation and secondly which is the definition of modernity supposed. Only after that, we can start with the historical analysis.

Insofar the article connects methodologically problem-centred theoretical considerations with historical-hermeneutical interpretations.

As a result, we can conclude that at the end of the 18th century, we can see the contours of a newly emerging social formation: namely, a functionally differentiated society. Processes of differentiation between different spheres of value and society were advanced at both the socio-structural and the discoursal level. While the Roman church preceded social development in the High Middle Ages by asserting its own theological rationality that could not be derived from society, over centuries tendencies towards self-assertion of secular spheres developed and at the end of the 18th century set the course for the emergence of the European modernity.
\end{abstract}

Keywords Functional differentiation - Religion · Emergence of modernity · Conflict · European modernity

Inwieweit der differenzierungstheoretische Ansatz das Potential besitzt, den Beitrag der Religion zur Herausbildung der europäischen Moderne verständlich zu machen, stellt die leitende Frage dieses Aufsatzes dar. Um die Frage nach dem religiösen Einfluss auf die Entstehung der modernen Welt differenzierungstheoretisch behandeln zu können, ist es zunächst erforderlich, das gebrauchte theoretische Instrumentarium zu erläutern. Es muss zum einen dargelegt werden, worin die wesentlichen Aussagen der Differenzierungstheorie bestehen, zum andern ist es notwendig anzugeben, welcher Begriff von Moderne vorausgesetzt wird. Eine sozialgeschichtliche Untersuchung mit einer so weitgehenden Fragestellung wie der hier aufgeworfenen muss, will sie sich nicht in den Tiefen der Geschichte verlieren, theoretische Gesichtspunkte bestimmen, von denen her die Analyse erfolgen soll. Und sie muss erläutern, was es denn ist, das sie sich vornimmt zu erklären, denn nur wenn das Explanandum definiert ist, lässt sich entscheiden, welchen historischen Abläufen und Ereignissen analytische Relevanz zukommt und welchen nicht.

Der vorliegende Aufsatz setzt ein mit einem Vorschlag zur soziologischen Bestimmung des Modernebegriffs (1.1.), nimmt dann eine problemorientierte Auseinandersetzung mit Grundannahmen der Differenzierungstheorie vor (1.2.) und untersucht schließlich in dem Versuch, soziologische Unterscheidungen auf geschichtliche Veränderungen anzuwenden, in großen historischen Schritten den Beitrag der Religion zur Emergenz der europäischen Moderne (2.). Der Aufsatz geht davon aus, dass der Durchbruch zur Moderne erstmals in Europa erfolgte. Zugleich stellt er in Rechnung, 
dass es unterschiedliche Pfade zur Herausbildung moderner Gesellschaften gibt, von denen der europäische nur einer unter mehreren ist. Nicht aber das Verhältnis dieser unterschiedlichen Wege in die Moderne stellt das Thema dieses Beitrags dar. Vielmehr geht es in ihm um die Emergenz der europäischen Moderne, die ohne den grundlegenden Einfluss des Christentums nicht zu verstehen ist. Zentrale Merkmale der europäischen Moderne, wie etwa horizontale und vertikale Differenzierung, bilden sich bereits im christlich geprägten Mittelalter heraus. Ihre Entstehung erfolgt in erster Linie - so die zentrale These dieses Aufsatzes -allerdings nicht durch die direkte Transformation christlicher Sinnformen in nichtchristliche, sondern vor allem durch den Konflikt mit den Geltungs- und Suprematieansprüchen des Christentums. Um den Beitrag des Christentums zur Herausbildung der europäischen Moderne besser erfassen zu können, wird daher hier der Vorschlag unterbreitet, den differenzierungstheoretischen Ansatz akteurstheoretisch und konfliktsoziologisch zu schärfen.

\section{Theoretische Überlegungen}

\section{1 Überlegungen zum Begriff und Konzept der Moderne}

Die Definition von konstitutiven Merkmalen moderner Gesellschaften wird in der Soziologie kontrovers diskutiert, und die Uneinigkeit hat sich noch einmal erhöht, seitdem mehr und mehr Soziologen dem Konzept der multiple modernities folgen (Eisenstadt 2000). Manch einer geht dabei so weit, die Definierbarkeit von Moderne überhaupt zu bestreiten und die Einheit der Moderne in ihrer Kontingenz zu sehen (Knöbl 2007). Demgegenüber bestehen die hier angestellten Überlegungen auf der Unausweichlichkeit einer soziologischen Bestimmung des Modernebegriffs, denn auch die Annahme einer Vielzahl von Modernen muss angeben, was sie unter Moderne versteht und worin die gemeinsamen Merkmale der unterschiedlichen Spielarten moderner Gesellschaften bestehen (Berger 2006, S. 203).

Im Unterschied zu philosophischen Ortsbestimmungen der Moderne, die oft nur ein Prinzip ins Zentrum rücken wie etwa Hegel in seiner Theorie der bürgerlichen Gesellschaft das Prinzip der freiheitlichen Subjektivität, bedienen sich soziologische Theorien der Moderne zur Erfassung von deren Spezifik zumeist einer Vielzahl von Kriterien. So zieht etwa Daniel Lerner (1968, S. 387) fünf Charakteristika der Moderne heran - ein sich selbst tragendes Wirtschaftswachstum, ein demokratisches Repräsentativsystem, die Verbreitung säkular-rationaler Normen, zunehmende Mobilität und die Ausprägung von empathischer Fremd- und individualistischer Selbstwahrnehmung - und Anthony Giddens (1996, S. 75 ff.) vier - Kapitalismus, Industrialisierung, Nationalstaat und das Machtmonopol des Staates.

Auch der hier vorzulegende Entwurf geht davon aus, dass sich moderne Gesellschaften aufgrund ihrer Komplexität und ihrer weit getriebenen Auflöse- und Rekombinationskapazitäten nicht einfaktoriell erfassen lassen, sondern zu ihrer Definition ein multiparadigmatischer Ansatz erforderlich ist. Vier Merkmale seien benannt. 
1. Mit den meisten vorliegenden Modernetheorien - von Weber, Simmel und Troeltsch, über Bourdieu und Giddens bis hin zu Luhmann - verfolgt das hier vertretene Konzept einen differenzierungstheoretischen Ansatz, demzufolge sich moderne westliche Gesellschaften durch das Prinzip einer weit getriebenen horizontalen Ausdifferenzierung gesellschaftlicher Sphären wie Recht, Wirtschaft, Wissenschaft, Politik, Kunst, Religion usw. auszeichnen. Differenzierung schließt Interdependenzen zwischen den einzelnen Teilbereichen und ihre wechselseitige Einflussnahme aufeinander nicht aus, sondern ausdrücklich ein. Auch wenn die Teilbereiche nicht unabhängig voneinander agieren, folgen sie intern jedoch selbst gesetzten Rationalisierungs- und Steigerungslogiken.

2. Die Herausbildung von unterschiedlichen gesellschaftlichen Sphären ist eng verbunden mit einem zweiten Differenzierungsprinzip: mit der vertikalen Auseinanderziehung gesellschaftlicher Konstitutionsebenen. Im Gegensatz zu vormodernen Gesellschaften greifen Herrschaftsinstitutionen und kulturelle Normen in modernen Gesellschaften nicht mehr unmittelbar auf die Lebensführung der Individuen durch. Vielmehr sind die soziale Makro- und Mikroebene über vielfältige, Freiraum gewährende Mechanismen miteinander vermittelt, wobei Märkten, formalisierten Verfahren und Organisationen eine zentrale Funktion zukommt. Die durch funktionale Differenzierung bedingte Herauslösung der Individuen aus der unmittelbaren Abhängigkeit von sozialen Milieus und sozialen Schichten und die dadurch bedingten Prozesse der Individualisierung gehen so Hand in Hand mit dem Ausbau formalisierter Organisationen, rechtlicher Bestimmungen und politischer Gelegenheitsstrukturen, über die sich „Gesellschaft“" auf die unterschiedlichen Formen der individuellen Lebensführung vermittelt (Beck 1983).

3. Die Dynamik moderner Gesellschaften beruht weniger auf Entlastungseffekten, die die einzelnen Funktionssysteme füreinander erbringen als vor allem auf der Einrichtung von Foren des Wettbewerbs, also von Märkten, innerhalb der einzelnen Funktionssysteme. Wettbewerbsforen, wie wir sie in der Wirtschaft, der Politik, der Wissenschaft, aber auch in der Schule und Ausbildung kennen, stellen einen Anreiz zur Leistungssteigerung und wechselseitigen Überbietung dar und sind entscheidende Motoren der gesellschaftlichen Dynamisierung. Unter Wettbewerbsbedingungen findet eine prinzipielle Delegitimation von Autoritäten, überkommenen Bindungen und Gewohnheiten statt, der sich kaum eine soziale Praxis entziehen kann. Letzte Ziele kann es in diesem Prozess permanenter Kritik und Veränderung nicht geben, weder in der demokratischen Willensbildung noch in der wissenschaftlichen Forschung oder in der ökonomischen Praxis. Die Moderne ist insofern kein Projekt, wie Habermas (1990a) behauptet, sondern ein prinzipiell ergebnisoffener Prozess.

4. Zugleich sind moderne Gesellschaften diesen Steigerungsprinzipien jedoch nicht hilflos ausgeliefert, sondern zur autonom begründeten Selbstbegrenzung fähig. Die Politik bindet sich an die Grundsätze der Gewaltenteilung und ordnet sich etwa der Rechtsprechung unter, Regierende erhalten ihr Mandat vom Souverän, dem Volk, und geben es auch wieder ab, wenn der Souverän anders entscheidet. Die Wissenschaft macht zu ihrem Erkenntnisgegenstand nur dasjenige, das sie mit Hilfe wissenschaftlicher Methoden erfassen kann und schränkt die Aussagekraft ihrer 
Erkenntnisse auf den Umkreis des methodisch gesicherten Wissens ein, erkennt also Grenzen seiner Zulänglichkeit an. Ebenso betreibt die Wirtschaft nicht Gewinnmaximierung um jeden Preis, sondern berücksichtigt auch die sozialen Bedingungen der Arbeiter und Angestellten, Kriterien der Arbeitszufriedenheit, ökologische Gesichtspunkte und Sicherheitsbedenken, sofern diese in Preise überführt werden können. Der modernen Steigerungsdynamik eignet also eine Tendenz zur reflexiven Selbstbeschränkung (Offe 1989).

Die sozial- und kulturgeschichtlichen Analysen zur europäischen Moderne, die in diesem Aufsatz angestellt werden, greifen hauptsächlich auf das differenzierungstheoretische Paradigma zurück. Da die dargestellten Merkmale moderner Gesellschaften miteinander zusammenhängen, wird aber gelegentlich auch auf die anderen drei Paradigmen Bezug genommen. Zur Erläuterung des differenzierungstheoretischen Ansatzes sollen zunächst seine Grundannahmen expliziert (1.2.1.) sowie Einwände gegen diese vorgestellt (1.2.2.) und diskutiert werden (1.2.3.).

\section{2 Überlegungen zum differenzierungstheoretischen Ansatz}

\subsubsection{Differenzierungstheoretische Grundannahmen}

Die soziologische Differenzierungstheorie hat seit Herbert Spencer (1882ff.) ihr Zentrum in der Prämisse, dass sich die Gesellschaft als Ganze im Laufe ihrer Geschichte differenziert (Nassehi 2004, S. 100), und sie verknüpft diese Prämisse mit der Behauptung, dass sich in diesem Differenzierungsprozess die Form der Differenzierung verändert, dass aus einer funktional diffusen eine funktional differenzierte Struktur entsteht (Tyrell 2008, S. 80 f.). In der am weitesten entwickelten Ausarbeitungsgestalt dieser Theorie, der Systemtheorie, wird die Emergenz der modernen Gesellschaft als Umbau der Gesellschaftsstruktur von Stratifikation auf funktionale Differenzierung gefasst, aufgrund derer sich unterschiedliche gesellschaftliche Funktionsbereiche - Recht, Wissenschaft, Ökonomie, Politik, Kunst - herauskristallisieren, die jeweils ihren eigenen Codes und Funktionsprinzipien folgen und zugleich interdependent aufeinander bezogen sind (Luhmann 1997). Im Unterschied zu vormodernen stratifizierten Gesellschaften laufe die moderne Gesellschaft nicht mehr auf eine das Ganze repräsentierende Spitze zu, sondern sei polyzentrisch strukturiert.

Interdependenzen und Austauschprozesse zwischen den gesellschaftlichen Bereichen sind von Formen der Entdifferenzierung zu unterscheiden. Entdifferenzierung bezeichnet den Einbau systemischer Fremdrationalität in ausdifferenzierte Sinnzusammenhänge (Gerhards 1991) und damit die Vermischung unterschiedlicher Sinnrationalitäten. Moralische Kriterien werden zur Beurteilung ökonomischer Kosten-/Nutzenabwägungen herangezogen, wissenschaftliche Wahrheitsansprüche politischen Interessen unterstellt, künstlerische Schönheitsideale mit religiösen Erlösungssehnsüchten verknüpft. Im Falle von Interdependenz, Ressourcentransfer und Austausch zwischen gesellschaftlichen Teilsystemen bleiben die Unterschiede zwischen den verknüpften Bereichen im Kern jedoch erhalten. 


\subsubsection{Einwände gegenüber dem differenzierungstheoretischen Ansatz}

Trotz seines prominenten Charakters - oder auch gerade wegen dieses Charakters - hat das Konzept der gesellschaftlichen Differenzierung eine Vielzahl von Vorbehalten, Kritiken und Zweifeln auf sich gezogen. Schon die Tatsache, dass es sich bei ihm um eine Langfristperspektive handelt, hat ihm den Vorwurf der Übergeschichtlichkeit und des Universalismus eingetragen (Joas 2014, S. 609f.). Kritische Einwände werden auch gegen den einlinigen, deterministischen und teleologischen Charakter des differenzierungstheoretischen Ansatzes vorgebracht. Der wichtigste Kritikpunkt aber richtet sich auf die mangelnde Erklärungskraft des Konzepts: Was sind die Ursachen von Prozessen sozialer Differenzierung, wer sind ihre Träger, wodurch wird Differenzierung gehemmt, wodurch befördert - das seien Fragen, die die Differenzierungstheorie nicht beantworte (Joas 1992, S. 326 ff.; Knöbl 2013, S. $99 \mathrm{ff}$.). Diese unterstelle zwar eine gesellschaftsweit ablaufende universelle Entwicklungstendenz, sei aber nicht in der Lage, die kausalen Mechanismen anzugeben, über die sich diese Tendenz reproduziert (Schimank 2005, S. 165 ff.).

Bezweifelt wird auch, dass funktionale Differenzierung überhaupt für moderne Gesellschaften typisch sei. Zeichnen sich moderne Gesellschaften nicht weniger durch die Ausdifferenzierung eigenlogischer Funktionssysteme aus als durch ihre Verzahnung und Interpenetration (Münch 1991)? Sind die Praktiken unterschiedlicher Funktionssysteme wirklich getrennt oder vollziehen sie sich fusioniert (Berger 2003, S. 210)?

In die entgegengesetzte Richtung geht eine Kritik, die sich nicht auf die differenzierungstheoretische Annahme der Entkopplung von Funktionen bezieht, sondern auf die differenzierungstheoretische Unterstellung eines interdependenten Zusammenhanges zwischen den sich ausdifferenzierenden Funktionsbereichen. Eine solche Annahme statte die Entwicklung der Funktionssysteme mit dem Merkmal wechselseitiger Abhängigkeit aus und unterschätze damit die Variabilität der Kombinationen, in denen die Gesellschaftsbereiche zueinander stehen können. Mit den Arbeiten Shmuel Eisenstadts (2000) zur Vielfalt der Moderne habe sich die Vorstellung von der Einheit der Moderne, in der die Ausdifferenzierung eines gesellschaftlichen Bereiches nicht ohne die gleichzeitige funktionale Spezifikation anderer Bereiche vonstattengehen könne, weitgehend erledigt (Schwinn 2006, S. 11).

\subsubsection{Diskussion der Einwände}

Um die Position zu skizzieren, von der her hier argumentiert wird, wollen wir uns auf drei der benannten Kritikpunkte konzentrieren: auf die Frage nach dem Sinn des Differenzierungskonzepts (1), auf die nach der Bestimmung des Verhältnisses von Differenzierung und Entdifferenzierung in modernen Gesellschaften (2) sowie auf die nach den Ursachen sozialer Differenzierung (3).

1. Soziologische Konzepte wie das der sozialen Differenzierung oder auch das der Rationalisierung oder Säkularisierung haben ihren Sinn nicht darin, dass sie sozialen Wandel in der Vielfalt aller seiner Aspekte abbilden. Sie fokussieren auf einen oder einige wenige Gesichtspunkte, um Vorher/Nachher-Unterscheidungen 
treffen zu können. Ihr Ziel ist es, anhand von konstant gehaltenen Merkmalen über längere Zeiträume sich erstreckende soziale Veränderungen zu erfassen. In der Soziologie dient die Theorie der sozialen Differenzierung vor allem dazu, moderne von vormodernen Gesellschaften abzugrenzen. Die Differenzierungstheorie ist also ein heuristisches Konzept, das Früheres und Gegenwärtiges unter einem Vergleichsgesichtspunkt in Beziehung setzt. Insofern ist Volkhard Krech (2014, S. 565) zuzustimmen, wenn er Prozessbegriffe als „Kondensate von Fragestellungen“ versteht. Was macht moderne Gesellschaften im Unterschied zu vormodernen aus? Kann der Begriff der funktionalen Differenzierung dazu beitragen, diesen Unterschied zu erfassen?

2. Moderne Gesellschaften zeichnen sich nicht nur durch Differenzierungs-, sondern auch durch Entdifferenzierungstendenzen aus. Differenzierung und Entdifferenzierung stehen jedoch - so die hier vertretene These - in einem asymmetrischen Verhältnis, in dem erstere gegenüber letzterer dominiert. Wodurch ist diese Dominanz bedingt? Natürlich sind die Grenzen der Funktionssysteme gegeneinander nicht fest geschlossen. Das wirtschaftliche System muss politische Eingriffe ebenso hinnehmen wie das Wissenschaftssystem, das wirtschaftliche System ist von moralischen Erwägungen so wenig frei wie die Medizin, und auch zwischen Religion und Medizin oder Seelsorge und Psychologie lassen sich Interferenzen beobachten. Wo die Grenzen zwischen den einzelnen gesellschaftlichen Bereichen jeweils verlaufen, ist umstritten. Zwar können Grenzen der gesellschaftlichen Teilbereiche dauerhaft nur von innen her aufgerichtet werden; ihre Bestimmung wird sowohl von innen als auch von außen jedoch immer wieder in Frage gestellt. Aushandlungsprozesse über die Gültigkeit von systeminternen Regeln und Leitwerten finden auf der Ebene von Organisationen, Märkten und Gemeinschaften, also nicht nur in horizontaler Hinsicht zwischen unterschiedlichen Systemen, statt, sondern auch zwischen Leistungsproduzenten innerhalb der jeweiligen Systeme selbst sowie zwischen Leistungsproduzenten und Leistungsempfängern und insofern auch vertikal. ${ }^{1}$

Wie die Kämpfe ausgehen, hängt von einer Vielzahl von Faktoren ab, wird letztendlich aber von einem simplen Anerkennungskriterium bestimmt. Es setzen sich diejenigen Regeln und Leitdifferenzen durch, die Resonanz finden, an die angeschlossen wird, auf die man sich beruft, auf denen man aufbaut und die man gegen Kritik vielleicht sogar verteidigt. Die Leitunterscheidungen konstituieren sich kumulativ (Nassehi 2004, S. 108), aber auch aversiv. Im Vollzug der Arbeiten ${ }^{2}$ an den Außengrenzen und an den internen Codes des ausdifferenzierten Feldes bilden sich immer wieder benutzte Kanäle und Austauschbeziehungen, man könnte sagen: Trampelpfade der sozialen Interaktion heraus, die sich durch wiederholte

\footnotetext{
1 Mit der Unterscheidung zwischen dem Verhältnis der Leistungsproduzenten eines Systems zu den Leistungsproduzenten anderer Systeme und zu ihren innersystemischen Konkurrenten und darüber hinaus zu den Leistungsempfängern wird ein Vorschlag Uwe Schimanks aufgenommen und modifiziert, den dieser vor einigen Jahren unterbreitet hat. Vgl. Schimank (2011).

2 Reuter (2009) spricht von „Grenzarbeiten am Feld“.
} 
Anschlusshandlungen und durch Abweisung von Abweichungen verstärken. ${ }^{3}$ Auf wissenschaftlichen Methoden beruhendes neues Wissen knüpft an methodisch hergestelltes altes Wissen an und sortiert methodologisch unqualifiziertes Wissen aus; Rechtsprechungen berufen sich auf Rechtsgrundsätze und bereits erfolgte Rechtsprechungen und sperren sich gegen rechtsfremde Gesichtspunkte; die marktinduzierte Bestimmung von Preisen reguliert Zahlungen und verhindert die staatliche oder moralische Beeinflussung von Preisen usw. Die ausdifferenzierten Codes und Prinzipien hören damit nicht auf, umstritten zu sein, und können immer wieder partiell außer Kraft gesetzt werden, gewinnen aber durch die kumulativen und aversiven Anschlusshandlungen eine relative, sich operativ wechselseitig bestärkende Stabilität. Dabei wird die sich in einer bestimmten Varianzbreite bewegende Regelkonformität auch dadurch verstärkt, dass regelwidrige Störungen und Interferenzen in die Unsichtbarkeit abgedrängt werden und Anschlusshandlungen an die sichtbare Seite des so Unterschiedenen anknüpfen.

3. Wie aber kommen Formen funktionaler Differenzierung im Laufe der Geschichte überhaupt zustande? Wie lassen sich Prozesse funktionaler Spezifikation erklären? Zwei mögliche und vielfach benutzte Erklärungen scheiden aus: der gesellschaftstheoretische Holismus, dem zufolge sich „die Gesellschaft“ selbst ausdifferenziert, so als wäre sie zunächst eine Einheit, die sich dann in sich selbst vervielfältigt. Preiszugeben ist aber auch die Erklärung funktionaler Differenzierung aus gesellschaftlichen Bedürfnissen und Erfordernissen. Nicht aufgrund von notwendig zu erfüllenden Funktionen in der Gesellschaft setzen Prozesse der funktionalen Differenzierung ein, denn auch wenn etwas wünschenswert oder unter bestimmten Gesichtspunkten vielleicht sogar erforderlich ist, kommt es deshalb noch nicht zustande. $^{4}$

Zur Lösung des differenzierungstheoretischen Erklärungsproblems schlagen Soziologen wie Uwe Schimank (1988), Thomas Schwinn (2001), Jens Greve, Clemens Kroneberg (Greve und Kroneberg 2011, S. 9 ff.), Hans Joas (1992, S. 336) und andere vor, der makrosoziologisch argumentierenden Differenzierungstheorie ein handlungstheoretisches Fundament zu geben. Diesem Vorschlag schließen sich die folgenden Überlegungen an.

Um die Analyse von Ursachen sozialer Differenzierungsprozesse voranzutreiben, erscheint es sinnvoll zu sein, trotz der unbestreitbaren Spontaneität des Handelns nach typischen und wiederkehrenden Mustern der Ausdifferenzierung zu fragen. Im Sinne von zu testenden Modellen seien hier drei Ausdifferenzierungsmechanismen unterschieden: Konfliktmechanismen (a), Transformationsmechanismen (b) und Selbstorganisationsmechanismen (c). Zum einen (a) scheinen Projekte der Entdifferenzierung, etwa von Religion und Politik oder auch von Religion und Wissenschaft

\footnotetext{
3 Vgl. bereits Durkheim (1992 [1893], S. 437), der die Entstehung integrierter gesellschaftlicher Strukturen über Grenzen hinweg darauf zurückführt, dass der Austausch regelmäßig stattfindet und sich häufig vollzieht, dass ,festumrissene Handlungsweisen [...] sich unter gegebenen Umständen als identische wiederholen“ und so zu Gewohnheiten, Bräuchen und Verhaltensregeln werden (ebd., S. 435). Der Austausch regele sich so „,von selbst, und mit der Zeit konsolidiert er sich vollends“ (ebd., S. 437). Es entstünden „Kanäle [...], die das Leben sich selbst gegraben hat“" (ebd., S. 435).

4 So wiederum bereits Durkheim (1970 [1895], S. 176f.).
} 
oder Religion und Recht, immer wieder Gegentendenzen der Entsynthetisierung des Zusammengeschlossenen zu provozieren. Projekte der Entdifferenzierung erschweren den Kompromiss, so dass sie, wenn sie aufeinanderstoßen, in aporetische Situationen führen, die sich oft nur noch lösen lassen, wenn das Zusammengeschlossene auf einer höheren sozialen Konstitutionsebene entflochten wird. Man könnte von Differenzierung aufgrund der Unlösbarkeit von Konflikten sprechen.

Ebenso (b) lässt sich beobachten, dass im Innern von entdifferenzierten Einheiten häufig die Keime von Differenzierungsprozessen angelegt sind, aus denen sich Neues entwickeln kann. Die Veränderung wird in diesem Falle nicht durch Konflikte bewirkt, sondern durch Transformation, durch Isolierung und Steigerung einzelner Elemente, durch ihre partielle Aneignung und Abstoßung, durch Verwandlung, Vereinseitigung und Uminterpretation.

Schließlich (c) können soziale Differenzierungen aber auch das Produkt von $e i$ gendynamischen Entwicklungen sein, die nicht durch Konflikte oder soziale Synthesen angestoßen, sondern durch sich ausweitende Gelegenheitsstrukturen ermöglicht werden. Diese bieten Raum für sich ausdifferenzierende neue Handlungszusammenhänge.

Im Rahmen der hier vorgelegten Analysen ist es ausgeschlossen, die heuristische Fruchtbarkeit aller drei unterschiedenen kausalen Mechanismen zu demonstrieren. Lediglich eine auf das Konfliktmodell rekurrierende These soll hier diskutiert werden. Dabei besteht die leitende Frage der folgenden Analysen darin, wie aus Konflikten, die aus integrativen Projekten der Entdifferenzierung entstehen, Formen der Differenzierung werden, in denen der grenzüberschreitende Entdifferenzierungsanspruch zurückgenommen und Zonen der Indifferenz zugelassen werden. Anders gefragt, wie wird aus einer konflikteindämmenden Hierarchie mit einer das Ganze repräsentierenden Spitze eine polyzentrische Struktur, wie sie für moderne Gesellschaften typisch ist. Die These der folgenden Abhandlung besagt, dass sich in diesem Umbau von hierarchischer Stratifikation zu polyzentrischer Differenzierung die Herausbildung der westlichen Moderne vollzieht und dass Konflikten, die aus Entdifferenzierungsansprüchen resultieren, dabei eine treibende Funktion zukommt. Der vorliegende Beitrag untersucht diese Prozesse der Modernisierung anhand von Differenzierungsschüben vom Hochmittelalter bis zum ausgehenden 18. Jahrhundert und die Rolle von Religion in diesen Prozessen.

\section{Differenzierungsschübe in der Herausbildung der Moderne: Eine kultur- und sozialgeschichtliche Analyse}

Unter der Voraussetzung, dass funktionale Differenzierung ein zentrales Merkmal moderner Gesellschaften ist, nimmt die hier zu vertretende These einen Bruch in den Formen der gesellschaftlichen Differenzierung zwischen Moderne und Vormoderne an und schenkt zugleich Antizipationen funktionaler Differenzierung in der Vormoderne eine besondere Beachtung. Der zentrale Bruch zwischen Vormoderne und Moderne lag, so die Behauptung, in der Zeit zwischen dem Ende des 17. und der ersten Hälfte des 19. Jahrhunderts. In dieser Periode, die weitgehend mit der von Reinhart Koselleck (1972: XIIIff.; 1989) als Sattelzeit bezeichneten Umbruchszeit 
übereinstimmt, wurde die dominante Differenzierungsform in den europäischen Gesellschaften von Stratifikation auf funktionale Differenzierung umgestellt. In dieser Periode wandelte sich die ständische zur bürgerlichen Gesellschaft, lockerte sich die Aufsicht der Kirchen über die Schule, erfolgte die Gründung konfessionsunabhängiger Universitäten, traten Kirche und Staat auseinander, löste sich die Kunst aus ihrer Abhängigkeit von Hof und Kirche, bildete sich eine standesübergreifende Öffentlichkeit heraus, entstand ein binnendifferenziertes System wissenschaftlicher Disziplinen und setzte sich der wirtschaftliche Markt von politischen Steuerungen ab. Schübe der funktionalen Differenzierung gab es auch in den Jahrhunderten davor, etwa im Hoch- und Spätmittelalter oder in der Reformationszeit, und danach, zum Beispiel in den langen 1960er Jahren. Die weitgehende Durchsetzung funktionaler Differenzierung als Strukturprinzip ganzer Gesellschaften indes war das Produkt der letzten zweihundert Jahre, und sie vollzog sich in dieser Radikalität ausschließlich im Westen.

Um 1800 setzte die great divergence (Pomeranz 2000) zwischen Europa und den Ländern Ostasiens ein. Parlamentarische Demokratien entstanden, das wirtschaftliche Wachstum erhöhte sich exponentiell, rechtsstaatliche Institutionen wurden aufgebaut, individuelle Freiheitsrechte schützende liberale Verfassungen verabschiedet, das Gewaltmonopol des Staates gesichert. In dieser Zeit traten die Wirtschaftsentwicklungen zwischen Westeuropa und den USA auf der einen und China und Indien auf der anderen Seite auseinander (Maddison 2007, S. 382). Allerdings gab es einen gewissen wirtschaftlichen Vorsprung Westeuropas auch schon in den drei vorangegangenen Jahrhunderten, der 1820 auf etwa das Doppelte im Vergleich zu China und Indien angewachsen war. Die great divergence hatte einen Vorlauf. Die hier vertretene These lautet denn auch, dass Prozesse der funktionalen Differenzierung bereits im Mittelalter ihren Ausgang nahmen und nach starken Gegenausschlägen vor allem in der Zeit des Konfessionalismus, in der nach den emanzipativen Impulsen der Reformation kirchliche und weltliche Ordnungen wieder weitgehend zusammenfielen, schließlich im 18. Jahrhundert zum Durchbruch kamen.

Doch wie lässt sich erklären, warum sich die Emergenz der Moderne gerade im 18. Jahrhundert und auf dem Boden des Okzidents vollzog? Es ist anzunehmen, dass neben einer Vielzahl von zusammenwirkenden Faktoren dem Christentum dabei eine zentrale Rolle zukam. Wahrscheinlich erhob keine andere religiöse Institution in der Weltgeschichte der Religionen jemals einen so weitreichenden Gültigkeits- und Gehorsamsanspruch wie das auf Autonomie und Allzuständigkeit insistierende römische Papsttum. Vermutlich hängt mit dieser Besonderheit zusammen, warum gerade auf dem Boden des lateinischen Christentums (und im Augenblick des Zusammenbruchs der Einheit dieser Christentumsgestalt) erstmals jene Strukturen entstanden, die wir für moderne Gesellschaften als charakteristisch ansehen.

\subsection{Vorläufer der funktionalen Differenzierung im europäischen Mittelalter}

Der hier zu entfaltenden These zufolge war es also das lateinische Christentum mit dem römischen Patriarchat an der Spitze, von dem der wesentliche Anstoß zur Ausdifferenzierung der gesellschaftlichen Sphären ausging. Mit ihrem Insistieren auf einer weltlich nicht ableitbaren, allein theologisch begründeten Rationalität ihres 
Handelns trieb die römische Kirche die anderen Bereiche der Gesellschaft dazu an, ebenfalls eigene Sachlogiken aufzubauen und diese gegen die religiöse Eigenrationalität in Stellung zu bringen.

Mit dieser These ist zunächst einmal die leitende Fragestellung des Lebenswerks von Max Weber aufgenommen, der gleichfalls fragte, ,,welche Verkettung von Umständen“ dazu geführt hat, ,dass gerade auf dem Boden des Okzidents, und nur hier, Kulturerscheinungen auftraten, welche doch - wie wenigstens wir uns gern vorstellen - in einer Entwicklungsrichtung von universeller Bedeutung und Gültigkeit lagen“ (Weber 1988 [1920], S. 1). Im Unterschied zu Max Weber soll hier allerdings nicht behauptet werden, dass die heutigen modernen Gesellschaften nur einen Ursprung haben und dieser im europäischen Westen lag und sich von dort aus universell verbreitete. Zur Moderne führen unterschiedliche Pfade. Vertreten werden soll hier lediglich die These, dass sich Strukturen moderner Gesellschaften das erste Mal in Europa herausbildeten, der Durchbruch zur Moderne also erstmals im Westen erfolgte.

Der Vorschlag, der Religion „die Rolle eines Vorreiters“ in der gesellschaftlichen Differenzierung zuzuweisen, stammt von Niklas Luhmann (1989, S. 260). Anders als Max Weber, mit dem er in der Herausstellung der religiösen Wurzeln der modernen Welt übereinstimmt, unternimmt Luhmann jedoch nicht den Versuch, die kapitalistische Wirtschaftsgesinnung und damit ein wesentliches Element des modernen Kapitalismus aus religiös geprägten Bewusstseinsstrukturen abzuleiten. Nicht Impulse einer religiös motivierten Arbeitsethik bildeten den Ausgangspunkt der Entstehung der Moderne, so Luhmann, sondern die strukturelle Ausdifferenzierung der Religion als ein ,prominentes, semantisch führendes Teilsystem der Gesellschaft", das den Aufbau seiner eigenen Rationalität vorantreibt und dadurch die anderen Lebensbereiche herausfordert, darauf mit Ablehnung zu reagieren und eigene Rationalitätslogiken zu konstruieren (Luhmann 1989, S. 344, 291, 260).

Die Anregung Luhmanns (1989, S. 261), Prozesse der funktionalen Differenzierung auf ,Sonderentwicklungen im Bereich der Religion“ zurückzuführen, ist inzwischen auch anderweitig aufgegriffen worden (vgl. Schneider 2011, S. 181; Schwinn 2013, S. 80f.). Sie muss in die Diskussion über die Ursprünge der Moderne im Mittelalter eingebettet werden, die sowohl von Historikern als auch von Soziologen und Rechtswissenschaftlern seit langem geführt wird (Nelson 1974; Strayer 1970; Southern 1995/2001; Berman 1991). Viele machen die Differenzierung von Königreichen, Fürstentümern, Städten, Gilden, Universitäten im 12. und 13. Jahrhundert als Wiege der modernen europäischen Gesellschaft aus (Nelson 1974, S. 30), andere hingegen den Investiturstreit mit seinen unmittelbaren Folgen bis hin zum Wormser Konkordat im 11. und 12. Jahrhundert (Böckenförde 2007 [1967], S. 49f.; Kaufmann 1989, S. 77 f.; Weinfurter 2006, S. 207). Unter differenzierungstheoretischen Gesichtspunkten kann die Verortung der Wurzeln der europäischen Moderne in die Zeit der zweiten Hälfe des 11. Jahrhunderts ein hohes Maß an historischer Plausibilität beanspruchen.

Im 11. Jahrhundert grenzte sich die römische Kirche im Zuge der Hildebrandschen Reformen strukturell aus den bestehenden politischen und sozialen Lebenszusammenhängen aus (1); sie entwarf sich als eine von der Welt unterschiedene autonome Organisation (2) und erhob trotz ihrer Abgrenzung von der Welt den 
Anspruch auf Superiorität über alle Bereiche der Gesellschaft (3). Mit dem Insistieren auf dem Zölibat, dem Verbot des Ämterkaufs und dem Kampf gegen die Investitur von Bischöfen durch weltliche Herrscher betrieb die Kirche ihre Selbstunterscheidung von der Welt (Tyrell 2010, S. 235) (1). Mit der Schaffung eines Kardinalskollegiums als dem einzig legitimen Gremium zur Wahl des Papstes und dem damit verbundenen Ausschluss von Volk und Laien aus dem Akt der Wahl legte sie sich auf einen selbstreferentiellen Rekrutierungsmodus fest. Der Stärkung ihrer organisatorischen Strukturen und ihrer Grenzen nach außen dienten auch die Einrichtung eines eigenen Verwaltungsstabes sowie die Konstitution einer selbständigen Jurisdiktionsgewalt (Dreier 2002, S. 4-6) (2). Mit der zugleich erhobenen Forderung nach Gehorsam nicht nur von allen Bischöfen und Priestern, sondern auch von Königen, Fürsten und Kaisern errichtete die römische Kirche schließlich einen Hegemonialanspruch über die ganze Welt. Nur der Papst sei wirklich universal. Nur ihm käme die Herrschaft über alle Bereiche des Lebens zu. Was er auf Erden binde oder löse, sei auch im Himmel gebunden oder gelöst. Dabei wurde seine prinzipielle Überordnung über alle weltlichen Entscheidungsgewalten aus seiner Einsetzung durch Gott hergeleitet: Als Statthalter Christi sei er auch das Haupt der Welt (3).

Mit der Aufrichtung eines solchen universellen Kompetenzanspruchs waren alle anderen gesellschaftlichen Akteure - Kleriker, Könige, Bauern, Gelehrte, Baumeister, Krieger ${ }^{5}$ - in den Status von Untergebenen oder Abweichlern gebracht. Funktionale Differenzierung wurde dadurch geradezu provoziert. Sie konnte sich nur gegen diesen Universalitätsanspruch entwickeln: als Abwehr von Suprematieansprüchen, als Bekundung von Nicht-Identifikation, als Dissens oder, wie es Hans Blumenberg sagt, als eine Form der humanen Selbstbehauptung. ${ }^{6}$ Selbstverständlich vollzogen sich Differenzierungsprozesse im Mittelalter im Rahmen eines allseits ge-

\footnotetext{
5 Das Mittelalter kennt bereits Formen einer Rollendifferenzierung. Während die Arbeitenden (laboratores $=$ Bauern) für die Ernährung zu sorgen hatten und ihnen ebenso wie den Betenden (oratores $=$ Geistlichkeit) das Recht auf Kriegsführung versagt war, hatten die Kämpfenden (bellatores= Adel) Frieden, Ordnung und Gerechtigkeit zu garantieren und waren wie die Kleriker von körperlicher Arbeit befreit (Oexle 1978, S. 25 f.; Tellenbach 1988, S. 108 ff.). Die Geistlichen wiederum hatten die Aufgabe zu beten, die Sakramente zu spenden und für das Seelenheil aller Menschen, einschließlich des Herrschers, zu sorgen (Erkens 2006, S. 72), aber es war ihnen verboten, Waffen zu tragen, richterliche Gewalt auszuüben, zu pflügen, zu jagen, Vieh zu halten, also die Arbeit der Bauern und die Aufgaben des Adels zu verrichten, denen wiederum die Verwaltung der Sakramente verwehrt war (Oexle 1978, S. 25 f.). Doch wurden diese Funktionsdifferenzierungen nicht konsequent durchgehalten. Die Bauern leisteten Kriegsdienst (Tellenbach 1988, S. 110 ff.), der hohe Klerus und der Papst führten Kriege (Hartmann 2014, S. 127), und der König hatte nach geltender Anschauung durch seine Krönung Anteil am Priesterkönigtum Christi und wurde so zum vicarius Christi (Hartmann 2014, S. 104). Das Reich wurde als eine religiöse Einheit gefasst. Trotz der Rollendifferenzierungen waren regnum, res publica, patria, sancta ecclesia, domus Dei austauschbare Begriffe (Weinfurter 2006, S. 30f., 37, 69, 75; Riches 2012). In welchem Verhältnis die Unterscheidung säkular - religiös zu den Rollendifferenzierungen stand, bedarf weitergehender Untersuchungen.

6 Blumenberg (1996) stellt die Entstehung der Neuzeit als einen Akt der Selbstbehauptung des Humanum gegenüber hypostasierten theologischen Absolutheitsansprüchen dar. Mit der Steigerung der Souveränität Gottes über alle menschlich fassbare Rationalitätskriterien im Nominalismus des Mittelalters, insbesondere mit der Überordnung des göttlichen Willens über seine Vernunft, treten Göttliches und Menschliches so weit auseinander, dass nur noch die Selbstbehauptung des Menschen gegenüber der potentiell allenthalben drohenden göttlichen Willkür bleibt. Diese von Blumenberg geistesgeschichtlich entwickelte Denkfigur wird hier sozialgeschichtlich gewendet.
} 
teilten christlichen Weltbildes. Auch wenn sich Kaiser wie Friedrich I. Barbarossa oder Friedrich II. gegen den Suprematieanspruch des Papstes stellten, taten sie dies stets unter Bezugnahme auf christliche Weltdeutungsmuster, indem sie sich etwa auf die Gottunmittelbarkeit ihres Königtums und Kaisertums beriefen oder die Notwendigkeit weltlicher Herrschaft aus der Schöpfungslehre ableiteten. Gleichwohl sind die Spannungen unverkennbar. Schon im Mittelalter gingen Denker wie Johannes Quidort von Paris (1255/60-1306), Dante Alighieri (1265-1321), Marsilius von Padua (gest. 1342/43) und Wilhelm von Ockham (1288-1347) von der legitimen Dualität von regnum und sacerdotium aus. Sie wiesen den weltlichen Herrschaftsanspruch der Kirche zurück und führten die weltliche Herrschaft auf nichtkirchliche Quellen zurück, sei es das Naturrecht, der Wille des Volkes oder das gegenüber dem Papsttum höhere Alter des Königtums.

Die Geschichte der funktionalen Differenzierung ist in ihren Anfängen also zunächst einmal eine Geschichte der Auseinandersetzung mit dem Totalitätsanspruch der römischen Kirche sowie mit der in ihr verkörperten Verschmelzung von weltlicher und geistlicher Herrschaft. Die Genese der modernen Welt kann mithin nicht als ein organisches Herauswachsen aus religiösen Anlagen verstanden werden. Dass ein wesentlicher Anstoß von der Kirche ausging, heißt nicht, dass sich religiöse Wurzeln in säkulare Konsequenzen transformiert hätten. ${ }^{7}$ Vielmehr wirkte der Impuls der römischen Kirche gerade in eine andere als die intendierte Richtung. Funktionale Differenzierung ist anfänglich in erster Linie eine Konfliktgeschichte. Sie kam als Ablehnung des kirchlichen Suprematieanspruchs auf den Weg. Aller Wahrscheinlichkeit nach hat die Singularität der westlichen Welt viel mit diesem Ausgangspunkt zu tun.

Die Abwehr des kirchlichen Suprematieanspruchs konnte konfliktiv erfolgen, aber wir finden im Hochmittelalter auch Fälle, in denen die Austragung des Konflikts zur Behauptung von Autonomie geradezu vermieden wurde. $\mathrm{Zu}$ denken wäre hierbei an die juristischen Diskurse der sogenannten Legisten. Diese grenzten sich zwar von den Kanonisten ab, bezogen sich aber niemals explizit auf deren Argumentationen, auch nicht negativ (Jansen 2019, S. 50). Die Kanonistik legte das kirchliche Recht aus, das sie auf die Bibel und die Schiften der Kirchenväter zurückführte, und verstand sich so als theologische Disziplin. Die Legisten hingegen griffen auf nichtkirchliche Quellen, auf römische Rechtstexte, den Codex iuris civilis zurück, den sie mit höchster, quasisakraler Autorität versahen (Jansen 2019, S. 47f.). Sie setzten so an die Stelle des kirchlichen ein säkulares Recht und etablierten sich damit als Juristen, die keine Theologen waren. Die von ihnen betriebene Ausdifferenzierung des Rechts verdankte sich dem Konflikt mit der Kanonistik und den hinter ihr stehenden Herrschaftsansprüchen der Kurie. Ihre Reaktion auf die Kanonistik bestand aber nicht darin, dass sie sie attackierten, sondern darin, dass sie sie bewusst ignorierten, sich ihr gegenüber indifferent verhielten, ihre Argumentation aus unabhängigen Quellen ableiteten und diese damit selbstreferentiell begründeten. Auch wenn der Konflikt Ausgangspunkt von Differenzierungsprozessen war, trieben die Legisten diese doch gerade dadurch voran, dass sie dem Konflikt auswichen

\footnotetext{
${ }^{7}$ Hier nehme ich die oben eingeführte Unterscheidung zwischen Konflikt und Transformation als unterschiedliche Ausdifferenzierungsmechanismen (neben dem Mechanismus der Selbstorganisation) auf.
} 
und ihn unsichtbar hielten. Mit der diskursiven Ausdifferenzierung einer weltlichen Rechtswissenschaft ging ihre Insitutionalisierung einher. An der mittelalterlichen Universität entstanden wissenschaftliche Gemeinschaften, die Studenten aus ganz Europa anzogen, zu Gelehrten ausbildeten und auf diese Weise eine hochgeachtete professionelle Juristenelite etablierten (Berman 1991, S. 260f.).

Wie wir sehen, waren die Prozesse der horizontalen Differenzierung, also der funktionalen Spezialisierung, von Beginn an mit Prozessen der vertikalen Differenzierung und damit mit Prozessen der Organisationsbildung eng verbunden. Das gilt für die Ausdifferenzierung des Rechtssystems ebenso wie für die Verselbständigungstendenzen politischer Herrschaft oder auch die vorangehende Abgrenzung der Kirche von der Welt. Indem die Kirche sich als von der Welt unterschiedene, selbstreferentiell begründete und weltüberlegene Instanz mit eigener Jurisdiktionsgewalt konstituierte, betrieb sie nicht nur die soziale Ausdifferenzierung des Religiösen, sondern auch dessen Institutionalisierung. Die Spezifikation des Religiösen erfolgte über die Ausbildung einer eigenständigen Organisation mit theologisch begründetem Programm, selbstreferentiellem Rekrutierungsmechanismus, hierarchischer Struktur und zweckrationaler Bürokratie. Auf diese Weise fiel der Start der funktionalen Ausdifferenzierung mit der Ausdifferenzierung einer eigenständigen Konstitutionsebene sozialer Kommunikation - der Ebene der Organisationsbildung - zusammen.

Neben den religiösen Suprematie- und Zuständigkeitsansprüchen der römischen Kirche und den durch sie ausgelösten politischen, wissenschaftlichen, rechtlichen und kulturellen Selbstbehauptungsbestrebungen trugen natürlich auch andere Faktoren zu den im Hochmittelalter einsetzenden sozialen Differenzierungsprozessen bei. Der wichtigste bestand in der relativen Schwäche der politischen Zentralgewalt, deren tatsächliche Wirkungsmöglichkeiten häufig hinter der von ihr beanspruchten Machtfülle zurückblieben. Das Reich - symbolisch repräsentiert durch den Kaiser überwölbte zwar die Territorien und stellte den rechtlichen und ideellen Ordnungsrahmen für die Herzog- und Fürstentümer dar, den diese weithin anerkannten. Nie gelang es jedoch dem Kaiser, den Eigenwillen der Territorien zu brechen und eine zentrale Herrschaftsstruktur durchzusetzen. Der Gegensatz zwischen Territorialund Zentralmacht blieb unaufgelöst. Auf der einen Seite leisteten die Herzöge dem König oder Kaiser den Treueeid, an den sie sich gebunden fühlten. Auf der anderen waren die Könige und Kaiser aufgrund des Königswahlrechts der Fürsten, aufgrund von Wahlversprechen (Wahlkapitulation) sowie aufgrund der ständischen Vertretungen, etwa auf den Reichstagen, von den Großen des Reiches abhängig und konnten nur im Zusammenwirken mit ihnen Politik betreiben. Die Bewohner waren nicht die direkten Untertanen des Königs oder Kaisers, sondern die Untertanen des jeweiligen Landesherrn. Auch dieser Umstand schränkte die unmittelbare Machtausübung der Könige ein. Hinzu kam, dass die königliche Macht durch die ständigen Auseinandersetzungen mit den nach Selbständigkeit strebenden Regionen Italiens und Burgunds sowie durch die Kriege mit den Nachbarn, vor allem mit Frankreich, militärisch gebunden und aufgrund der territorialen Überdehnung ökonomisch, finanziell, militärisch und personell überfordert war. Die immer wieder eskalierenden Konflikte mit der machtbewussten römischen Kirche forderten die Kräfte der kaiserlichen und königlichen Macht zusätzlich heraus. 
So vermochte die politische Zentralgewalt des europäischen Mittelalters weder autonom noch imperial zu agieren. In partieller Abhängigkeit von selbstbewussten Herzogtümern, in ständiger Abwehr päpstlicher Dominanzansprüche sowie im angestrengten Bemühen um Erhalt und Ausdehnung ihrer Macht war diese in einen Mehrfrontenkampf verwickelt, der sie daran hinderte, auf die Territorien, die Städte, den Adel, die Handwerker, die Bauern, die Universitäten, die Gelehrten und die Kleriker unmittelbar durchzugreifen. Zwar konnte das Reich dem Handeln, Denken und Wahrnehmen der Menschen einen rechtlichen und ideellen Ordnungsrahmen bieten, aber es musste ihnen doch auch einen weitgehenden Freiraum lassen, der die Entfaltung ihrer vielfältigen Handlungsinteressen begünstigte.

Zusammengefasst lässt sich also sagen: Der Superioritätsanspruch des römischen Episkopats forderte die unterschiedlichen Bereiche der Gesellschaft - Recht, Politik, Scholastik, Kunst, Literatur - und sogar die Akteure des geistlichen Lebens (Theologen, Mönche, Nonnen, religiös engagierte Bürgerinnen und Bürger) zur Selbstbehauptung heraus. Dass sich die unterschiedlichen Bereiche der Gesellschaft ausdifferenzierten, lag aber nicht nur an diesem Hegemonialanspruch, sondern auch daran, dass die Kontroll- und Steuerungsmöglichkeiten der politischen Zentralgewalt eingeschränkt waren und politische Freiräume und Gelegenheitsstrukturen existierten. Für das Anlaufen funktionaler Differenzierung ist mithin beides von Bedeutung: dass es eine religiöse Universalinstitution mit umfassendem Überlegenheitsanspruch gab, die Tendenzen der Ausbildung von Formen einer nichtreligiösen Rationalität geradezu erzwang, und dass eine politische Zentralinstanz fehlte, die in der Lage gewesen wäre, derartige Autonomiebestrebungen niederzuhalten und deren Ressourcen entweder zu vernichten oder einzugemeinden. So erwies sich die unauflösbare Dualität von geistlicher und politischer Macht in sozialer, kultureller und institutioneller Hinsicht letztendlich als ein produktiver Faktor, wenn nicht als der produktive Faktor für die Herausbildung der modernen Welt. ${ }^{8}$

Der nächste Differenzierungsschub, auf den hier ausführlicher einzugehen ist, vollzog sich in der sogenannten Sattelzeit, also im langen 18. Jahrhundert. Die beiden Jahrhunderte davor waren durch starke Entdifferenzierungstendenzen gekennzeichnet. Von der Reformation Martin Luthers gingen zwar zunächst Impulse zu einer schärferen Trennung von Religion und Politik aus, mit der Politisierung der Reformation, die bereits zu Lebzeiten Luthers einsetzte, schwächten sich diese Impulse jedoch ab. Die Geschichte der Differenzierung lässt sich nicht als ein einliniger Prozess verstehen; vielmehr müssen in die Rekonstruktion der Differenzierungsprozesse die gegenläufigen Bewegungen und retardierenden Tendenzen stets mit einbezogen werden.

\footnotetext{
8 Die Tragfähigkeit dieser These ließe sich nur durch interkulturelle Vergleiche überprüfen, die jeweils nach der Dominanz der politischen Zentralgewalt über andere gesellschaftliche Sphären, nach der Reichweite des Geltungsanspruchs religiöser Institutionen, nach dem Grad der Trennung des Religiösen vom Säkularen usw. zu fragen hätten. Derartige Vergleichsstudien würden den hier gesteckten Rahmen allerdings überschreiten.
} 


\subsection{Das Konfessionelle Zeitalter}

Den Ausgangspunkt für Luthers Drängen auf Trennung von Religion und Politik bildete seine Unterscheidung zwischen dem inneren und dem äußeren Menschen, zwischen Leib und Seele, wie er sie in seinem Traktat „Von der Freiheit eines Christenmenschen“ entfaltete (Luther 1520). In seinem Innern, seinem Gewissen, gehöre der Christ dem Reiche Gottes an und könne durch keine äußere Gewalt zum Glauben gezwungen werden. Im Reich der Welt wiederum will Luther das geistliche Regiment, das durch die Evangeliumsverkündigung der Ausbreitung des Reiches Gottes diene, und das weltliche Regiment, das angesichts der Macht der Sünde in dieser Welt für Ordnung und Frieden zu sorgen habe, getrennt sehen (Strohm 2008, S. 223f.). Die Evangeliumsverkündigung geschehe ohne Gewaltanwendung, allein durch das Wort. Das weltliche Handeln aber bediene sich des Schwerts. Es könne sich nur auf den äußeren Menschen beziehen, nicht aber auf Fragen der Gesinnung und des Glaubens.

Aus dieser Ansicht folgte für Luther, dass falsche Lehren nicht durch Anwendung von Gewalt überwunden werden können, sondern allein durch die Verkündigung des Evangeliums, durch das Wort, das die Herzen ergreift. „Ketzerei ist ein geistlich Ding, das kann man mit keinem Eisen zerhauen, mit keinem Feuer verbrennen, mit keinem Wasser ertränken.“ (Luther 1523, S. 268) Die Trennung von weltlichem und geistlichem Regiment ist mithin erforderlich, um den Glauben von allem äußeren Zwang freizuhalten. Sie ergibt sich direkt aus Luthers Eintreten für die Autonomie des gläubigen Individuums.

Das theologisch begründete Prinzip der Trennung von Religion und Politik konnte jedoch kaum praktische Bedeutung erlangen, denn zur Rettung der Reformation war Luther auf den Schutz der Fürsten angewiesen. Deshalb bat Luther den sächsischen Kurfürsten um die landesherrliche Aufsicht über die Kirche (Wallmann 2012, S. 62 f.). Obwohl er ursprünglich damit nur eine Übergangs- und Notlösung im Auge gehabt hatte, war auf diese Weise die anfängliche klare Trennung von geistlichem und weltlichem Regiment wieder aufgehoben. Den Verzicht auf die gewaltsame Verfolgung von Ketzern ließ Luther prinzipiell zwar unangetastet, aber in einer gewissen Unausgeglichenheit mit diesem Prinzip nahm er in Situationen ,problematischer religiöser Devianz" (Stegmann 2014, S. 484) das ordnende Handeln der Obrigkeit gleichwohl in Anspruch, etwa im Zusammenhang mit den während seines Aufenthalts auf der Wartburg eingeführten kirchlichen Neuerung in Wittenberg 1521/22, im Zusammenhang mit den Bauernaufständen von 1525 oder auch gegenüber den Schwärmern, Rottengeistern und Wiedertäufern sowie gegenüber den Juden, die er mehr und mehr als bekehrungsunwillig ansah.

Sofern an einem Ort zweierlei Lehren gepredigt würden, müsse die weltliche Obrigkeit darauf zu ,achten, dass nicht Zwietracht, Spaltungen und Aufruhr sich unter den Untertanen erheben“ (1528, S. 72). Auch wenn ihr nicht geboten sei, ,zu lehren und geistlich zu regieren", sei es ihr um der Wahrung von Ordnung und Sicherheit willen erlaubt, für die wahre Lehre zu sorgen.

Melanchthon ging in dieser Hinsicht noch einen Schritt weiter. Er sah die Verantwortung weltlicher Fürsten für den Erhalt des wahren Glaubens nicht nur in Fällen von Uneinigkeit, Zwietracht und Abweichung als eine gebotene Pflicht, sondern ge- 
nerell als theologisch gerechtfertigt an. Da die weltliche Macht dem Ruhme Gottes nur dienen könne, wenn es in ihrem Geltungsbereich keine gegen den wahren Glauben gerichteten Formen der Religionsausübung gebe, besitze sie für den Glauben ihrer Untertanen eine religiöse Fürsorgepflicht (Leonhardt 2017, S. 146f.). Eingeführt zunächst nur aus pragmatischen Gründen zur Behebung einer kirchlichen Not, wurde die Übertragung kirchlicher Aufgaben an den Landesherrn so schließlich zum Normalfall.

Nachdem in der Reformation die Einheit der mittelalterlichen Kirche zerbrochen war, legte der Augsburger Religionsfrieden fest, dass jeder Landesherr das Recht habe, über die Religion seiner Untertanen zu bestimmen. Zugleich erkannte er für das Reich im Ganzen die unterschiedlichen Bekenntnisse an. Der Augsburger Religionsfrieden zog also eine Differenzierung der Ebenen nach sich. Auf der Reichsebene wurde die religiöse Wahrheitsfrage gleichsam eingeklammert und ein religionsneutrales Reichsrecht oberhalb der konkurrierenden religiösen Wahrheitsansprüche etabliert (Heckel 2007, S. 13). Auf der Ebene der Territorien hingegen sollte nach dem Grundsatz „Cuius regio eius religio“ die religionspolitische Einheit gewahrt bleiben. Die Suspendierung der Wahrheitsfrage bedeutete indes nicht, dass die Wiedervereinigung der Konfessionen als Fernziel aufgegeben worden wäre. Die „endliche Vergleichung“ der Konfessionen wurde vielmehr als der eigentliche Zweck des Religionsfriedens festgehalten (Buschmann 1994, S. 221, Augsburger Reichsabschied § 9). Die Religionseinheit wurde jedoch insofern dem politischen Ziel der Friedenssicherung untergeordnet, als die Friedenspflicht auch dann geboten blieb, wenn die Überwindung der Glaubensdifferenzen misslingen sollte (Buschmann 1994, S. 229, Augsburger Reichsabschied § 25). Die Anerkennung der Religionsdifferenz war folglich die Bedingung für die Wahrung des politischen Friedens, und ohne diesen konnte auch die angestrebte religiöse Einheit nicht erreicht werden.

Was auf der Reichsebene preisgegeben worden war, sollte auf der territorialen Ebene erhalten bleiben: die Einheit von Religion und Politik. Nicht nur nach katholischer, sondern auch nach reformatorischer Lehre galten Recht, Staat und Obrigkeit als göttliche Stiftungen. Auch wenn die lutherische Zweiregimentenlehre den politischen Herrscher nur für die Ordnung der Welt als zuständig ansah, kam ihm doch die Fürsorgepflicht für die Durchsetzung der reinen Lehre zu (Sehling 1914, S. 8). Zur Bewahrung göttlichen Wohlwollens trug die christliche Obrigkeit Verantwortung für die Sicherung der christlichen Praxis und die Einhaltung der Gebote (Otto 2016, S. 38), denn das Verhalten des Einzelnen vor Gott galt als ausschlaggebend nicht nur für das Ergehen des Einzelnen, sondern auch für das Wohl und Wehe des Gemeinwesens. Deshalb übte die Obrigkeit in dieser Zeit - unter anderem über die Kirchenzucht, die der Staat im Auftrag der Kirche zur Sicherstellung der kirchlichen Ordnung und Lehre wahrnahm ${ }^{9}$ - eine scharfe Kontrolle über den Glauben ihrer Untertanen sowie über alle Aspekte ihrer religiösen Lebensführung aus (StollbergRilinger 2006, S. 94). ${ }^{10}$

\footnotetext{
9 Das römisch-katholische Äquivalent bestand in der 1542 gegründeten Kongregation für die Glaubenslehre.

10 Die neuere Forschung (Schindling 1997; Siebenhüner 2007, S. 259-262; Stollberg-Rilinger und Pietsch 2013) legt den Akzent weniger auf die Frage nach der konfessionellen Durchdringung der sozialen und
} 
Ebenso wie die politische Herrschaft als göttlich legitimiertes Regiment religiöse Aufgaben zu erfüllen hatte, übernahm die Kirche herrschaftliche Funktionen. Aufgrund ihrer Allianz mit dem frühmodernen Staat agierte die Kirche im konfessionellen Zeitalter in Stadt und Land nicht selten als dessen Repräsentant. Über Predigt, Beichte und Seelsorge sowie die Verbreitung von Andachts-, Gebets- und Gesangbüchern übte sie einen unmittelbaren Einfluss auf die Ausbildung von Normen im Glauben, Denken und Verhalten der Menschen aus. Auf diese Weise war sie im gesellschaftlichen Leben, in Politik, Rechtsprechung, Erziehung und Familie allgegenwärtig. Kirche und Staat, Predigtamt und Schule, Glaube und Familie durchdrangen sich wechselseitig (Schlögl 2013, S. 28 ff.; Schilling 1988, 2009; Dinges 1991). Auch wenn die Einheit der mittelalterlichen Kirche zerbrochen war, kamen so im Zeitalter des Konfessionalismus starke Tendenzen der Entdifferenzierung von Religion und Politik sowie von Religion, Recht und Gesellschaft zum Tragen, nur eben nicht mehr im gesamten Reich, sondern in den einzelnen Territorien.

Die mittelalterliche Vorstellung, dass die Staatsgewalt eine göttliche Stiftung sei, dass jede Abweichung von der staatlich anerkannten Religion die Legitimität der Staatsgewalt in Frage stelle, dass es selbstverständlich nur eine Glaubenswahrheit geben könne und dem Herrscher daher die Pflicht zukomme, den wahren Glauben politisch durchzusetzen, war im konfessionellen Zeitalter also nicht preisgegeben. Der Unterschied zur vorreformatorischen Epoche bestand darin, dass sich keine Einigkeit mehr darüber erzielen ließ, welches denn nun die wahre Kirche sei. Das Bemühen um die Wiederherstellung der politischen Einheit wurde daher als unerbittlicher Streit um die Dominanz des jeweils eigenen Bekenntnisses ausgetragen. Indem sich die katholische Kirche im Tridentinum auf die Vulgata, die lateinische Bibelübersetzung des Hieronymus, als authentischen Bibeltext festlegte, den Sakramenten göttliche Qualität zusprach, die Lehre von der Rechtfertigung des Sünders allein durch den Glauben verwarf und die Zentralstellung des Papstes stärkte, stellte sie sich der Reformation entgegen ${ }^{11}$ und hielt sie zugleich an dem Anspruch fest, die wahre Kirche Christi zu sein. Mit dem Jesuitenorden entstand eine geistliche Organisation, die mit der gleichen Unbedingtheit wie die Reformatoren für ihre theologischen Überzeugungen eintrat, durch Missions- und Erziehungsarbeit politischen und gesellschaftlichen Einfluss zu gewinnen versuchte und sich energisch von abweichenden theologischen Positionen abgrenzte. Indem das Luthertum vermehrte Anstrengungen unternahm, das Wort Gottes als Grundlage der eigenen theologischen Lehre zu befestigen und den Wortlaut der Bibel als göttlich inspiriert auszuweisen, vertiefte es seinerseits die Gräben. Die einander bekämpfenden religiösen Parteien stimmten zwar darin überein, dass nur eine einzige Konfession universale Gültigkeit besitzen könne und dass das Reich der religiösen Einheit bedarf. Gerade deshalb nahm der Streit jedoch einen unerbittlichen Charakter an.

politischen Ordnung als auf die Überschreitung konfessioneller Grenzen und die dadurch bedingte Relativierung, Einebnung und Vermischung konfessioneller Zugehörigkeiten. Siebenhüner (270) meint sogar, dass bei einer Beachtung der Grenzüberschreitungen die Frage nach dem Erfolg oder Misserfolg der Konfessionalisierung an Relevanz verliere.

11 Anders Wassilowsky (2016), der das Tridentinum nicht als ein geschlossen anti-protestantisches Bollwerk betrachtet wissen will. 
Der Kampf um den rechten Glauben, der dieses Zeitalter der kirchlichen Zerrissenheit, der religiösen Überhitzung und der normativen Enge kennzeichnete, führte bis zur gewaltsamen Verfolgung der Andersgläubigen. Die Bartholomäusnacht von 1572 in Paris, in der Tausende von Calvinisten blutig ermordet wurden, die Verbrennung des spanischen Humanisten Michel Servet 1553 als Ketzer im calvinistischen Genf und andere von unterschiedlichen Seiten verübte Gewalttaten stehen für diese religiöse Überhitzung. Die massenhaften Hexenverfolgungen, die ihren Höhepunkt nicht etwa in der Zeit des vorreformatorischen Mittelalters und im Spanien der Inquisition, sondern im konfessionell gespaltenen Reich erreichten, sind ebenfalls ein Ausdruck der das konfessionelle Zeitalter kennzeichnenden normativen Verfestigung. Und auch die Stimmen der Moderation, wie etwa die von Sebastian Castellio oder die der Sozianer, künden in ihrem auf Ausgleich bedachten Bemühen noch vom religiösen Eifer der Zeit.

Aus der engen Verknüpfung von Religion und Politik erklärt sich nicht nur die politische Relevanz religiöser Diskurse im Konfessionellen Zeitalter, sondern auch, warum viele der politischen Streitigkeiten im Medium der Religion ausgetragen wurden. ${ }^{12}$ Zwar gab es bereits einige gesellschaftliche Bereiche, die einer von Kirche und Religion weithin unberührten Logik folgten - man denke etwa an die Kunstmärkte im nördlichen Italien, an die Aufnahme des spätantiken Römischen Rechts, des Codex Justinianus, in die Rechtsprechung oder an die Eigenständigkeit des Finanzsektors -, im Großen und Ganzen behaupteten religiöse Argumente aber noch den Primat im gesellschaftlichen Diskurs und konnten sich im Konfliktfalle - das zeigt der Fall des Galileo Galilei - mit institutioneller Unterstützung gegen widerstreitende Gesichtspunkte auch immer wieder durchsetzen. Das änderte sich etwa ab Mitte des 17. Jahrhunderts.

\subsection{Das Aufkommen quasi-religiöser Konkurrenzansprüche seit dem 17. Jahrhundert und die Verschärfung der religionsinternen Pluralisierung}

Nach den verheerenden Erfahrungen der Religionskriege, die den konfessionellen Enthusiasmus verzehrt und den religiösen Fanatismus diskreditiert hatten, drängten zunehmend nicht-religiöse und teilweise sogar anti-kirchliche Tendenzen in den Vordergrund. Besonders deutlich wurde das in der Stärkung des frühneuzeitlichen Staates, der das Gewaltmonopol für sich beanspruchte, ständische Beschränkungen aufzuheben suchte und sich über konfessionelle Geltungsansprüche stellte. Das Blutvergießen des Dreißigjährigen Krieges hatte deutlich gemacht, dass das Allgemeinwohl über den von den Konfessionen verfochtenen Absolutheitsansprüchen

\footnotetext{
12 Die Forschung ist sich weitgehend darin einig, dass es sich beim Dreißigjährigen Krieg bis 1630 um einen ,,vorwiegend aus religiöser Motivation geführten Krieg, einen Religionskrieg“ gehandelt hat (Brendle 2011, S. 501). In der Einschätzung der Zeit, die sich von dem historiographischen common sense unterscheidet, waren sowohl die protestantische als auch die katholische Konfessionspartei auch nach dem Eingreifen Schwedens und Frankreichs noch davon überzeugt, in einem Religionskrieg zu stehen (ebd., 502). Das Eingreifen Gustav Adolfs wurde in erster Linie als religiös motiviert empfunden. Gerade der Eintritt der europäischen Mächte Frankreich und Schweden in den Krieg hatte zur Folge, dass Katholiken und Protestanten nun glaubten, die endgültige Entscheidung um den wahren Glauben stehe unmittelbar bevor.
} 
stehen muss: Salus rei publicae suprema lex est. ${ }^{13}$ Insofern hat der Dreißigjährige Krieg zur Befreiung des Staatswohls von der Religion, zur Zurückweisung religiöser Superioritätsansprüche und zur Überordnung des Politischen über das Religiöse beigetragen.

Auch die Staatslehren des 17. Jahrhunderts waren eine direkte Reaktion auf die Grausamkeiten und Wirren der blutigen Religions- und Bürgerkriege im konfessionellen Zeitalter. Deutlich wird das bereits an dem Ansatz des bedeutendsten Staatstheoretikers der damaligen Zeit: Thomas Hobbes. Der von ihm fiktiv unterstellte Naturzustand, in dem der Krieg eines jeden gegen jeden herrsche und der Mensch dem Menschen ein Wolf sei, glich der erfahrenen Anarchie des Bürgerkrieges. Nur ein souveräner Staat, der jede Unbotmäßigkeit rücksichtslos unterdrücke und keine irdische Instanz, auch nicht das Volk, über sich dulde, könne diesen Zustand des kriegerischen Chaos überwinden. Die Untertanen hätten den staatlichen Anordnungen vorbehaltlos zu folgen. Indem der Einzelne im Akt des Vertragsschlusses seine Macht an den Staat delegiere, habe er das Recht verwirkt, von diesem noch Rechenschaft für sein Handeln zu verlangen. Darin bestand die Bedingung für die Befriedung des Landes und für die Wiederherstellung der durch religiösen Wahrheitseifer zerstörten menschlichen Ordnung: dass die politische Autorität über der religiösen Wahrheit steht. Auctoritas non veritas facit legem. Mit diesem Satz schrieb Hobbes dem souveränen Staat eine Autorität zu, die im Mittelalter allein Gott besaß.

Am konsequentesten wurde diese Staatsdoktrin im katholischen Frankreich Ludwig XIV. verwirklicht. Abweichungen von der herrschenden Kirche seines Landes unterdrückte Ludwig XIV. nach Kräften. Seine Rekatholisierungspolitik folgte dem Grundsatz „Ein König, ein Gesetz, ein Glaube“. 1685 hob er das Edikt von Nantes auf, das den Hugenotten religiöse Toleranz und relativ freie Religionsausübung gewährt hatte. Hunderttausende wanderten in die europäischen Nachbarländer aus. Wer nicht auswanderte, musste mit Zwangsbekehrungen und blutigen Verfolgungen rechnen. Obwohl das Regime von Ludwig XIV. und seinen Nachfolgern absolutistische Züge besaß, wäre es, wie die neuere Forschung zeigt, jedoch unangemessen, es mit dem Begriff des Absolutismus zu belegen. Die Krone versuchte zwar, die Beharrungskraft der Stände, der ständischen Korporationen und der Gerichtshöfe (,,parlements“) zu brechen, scheiterte mit ihren Versuchen zur Modernisierung von Verwaltung, Justiz und Steuerwesen dann aber doch immer wieder an deren Widerstand (Stollberg-Rilinger 2006, S. 195). Auch gingen die Rationalisierungsbemühungen der Zentralgewalt mit aufklärerischen Bestrebungen der Philosophen, Juristen, Dienstadligen und Klerikern oft Hand in Hand. Jedenfalls gelang es den in Opposition zur Krone stehenden Aufklärern vielfach selbst im zentralistischen Frankreich, Schlüsselpositionen in Verwaltung und Regierung einzunehmen (Stollberg-Rilinger 2006, S. 196).

Dabei ist die enge Verbindung von Kirche und Staatsgewalt, wie sie auch noch für die Jahrhunderte nach dem konfessionellen Zeitalter typisch war, in erster Linie nicht mehr als eine Form der Diffusion von Religion und Politik anzusehen, sondern vor allem als eine Form der Indienstnahme der Religion durch die Politik. Wenn

${ }_{13}$ Mit diesen Worten drückte ein vornehmer Kölner das Resultat des Dreißigjährigen Krieges gegenüber dem uneinsichtigen Glaubenskämpfer von Wartenberg aus (Brendle 2011, S. 507). 
Ludwig XIV. die Unabhängigkeit der französischen Nationalkirche stärken wollte, verbündete er sich mit dem höheren Klerus gegen Rom; verfolgte er das Ziel, die Jansenisten zu bekämpfen, nahm er die Unterstützung Roms hingegen durchaus in Anspruch. In anderen katholischen Staaten, etwa im Habsburger Reich, waren die Jansenisten mit ihrer Überordnung der Gesamtheit der Gläubigen über den Papst die natürlichen Bündnisgenossen der katholischen Regierungen, um sich gegen die Interventionen Roms und die Gewährung von Sonderrechten für die katholische Kirche in ihrem Staatsgebiet zur Wehr zu setzen. Seit der Mitte des 17. Jahrhunderts bestimmten zunehmend politische Interessen das herrschaftliche Handeln, kaum noch religiöse.

Diese Auffassung vertritt auch Ernst-Wolfgang Böckenförde (2007 [1967], S. 57-61), der bereits bei den französischen Kronjuristen im 16. Jahrhundert, den sogenannten Politiques, die Tendenz beobachtet, Religion und Politik zu trennen und den Konfessionalismus politisch einzuhegen. Bestätigt sieht Böckenförde diese Tendenz bei Richelieu im 17. Jahrhundert, der in seinem politischen Testament die „raison“ zur obersten Richtschnur des politischen Handelns erklärt und einen religiösen Bezug nur noch insofern herstellt, als er die menschliche „raison“ als von Gott erschaffen behandelt. Mit der Einrichtung des „Parlaments der Heiligen“ unter Oliver Cromwell wurde das letzte Mal auf gesamtstaatlicher Ebene in Europa der Versuch unternommen, Politik mit religiösen Mitteln zu betreiben. Seitdem war die Religion der Politik untergeordnet, auch wenn sie sich mit ihr verbündete. Sie wurde zu einer staatlich kontrollierten Macht im europäischen Staatensystem. Der Staat konnte sich ihrer bedienen und zu seiner Unterstützung heranziehen, auf ihn einen dominanten Einfluss auszuüben, vermochte sie nicht mehr. Dabei stellt Böckenförde (2007 [1967], S. 55f.) die These auf, dass die Behauptung der Suprematie der Politik auf die Erwartung der geistlichen Macht gegenüber der weltlichen Macht, für die Durchsetzung der religiösen Wahrheit zu sorgen, und die sich daraus ergebenden permanenten politischen Konflikte zurückzuführen ist. Wollten die Fürsten sich nicht zu Exekutionsbeamten religiöser Interessen machen, mussten sie um der Wahrung der politischen Ordnung willen die geistlichen Dinge selbst in die Hand nehmen und den Primat der Politik gegenüber der Religion zur Geltung bringen. Sollte diese These richtig sein, wäre der Anspruch auf politische Autonomie auch hier wieder religiös erzwungen.

Doch nicht nur die Politik setzte sich von der Religion ab und stellte sich über sie. In den Bereichen von Recht, Naturwissenschaft, Philosophie, Erziehung, Kunst, Moral lassen sich vergleichbare Tendenzen beobachten. Nachdem die Religion aufgrund ihrer internen Zersplitterung in unterschiedliche Bekenntnisse als normative Grundlage diskreditiert war, begann eine vielfältige Suche nach einem neuen tragfähigen kulturellen Fundament. Diese neuen Bestrebungen nahmen die religiösen Semantiken und Argumentationsfiguren in unterschiedlicher Weise auf, beriefen sich auf sie und legitimierten sich durch sie. Ihr Bemühen aber war nun zunehmend darauf gerichtet, sich von religiösen Begründungen loszulösen, sich auf eigene Füße zu stellen und die religiösen Legitimationen überflüssig zu machen. ${ }^{14}$

\footnotetext{
${ }^{14}$ Sie zielten auf Autonomie und standen insofern zueinander im Verhältnis sich wechselseitig überbie-
} tender Konkurrenz. Die Epoche von der Mitte des 17. bis zum Beginn des 18. Jahrhunderts könnte man 
Das wird schon in dem philosophischen Versuch René Descartes' zur metaphysischen Neubegründung der Epistemologie deutlich. Descartes beginnt seine Überlegungen mit dem grundsätzlichen Zweifel an allen überkommenen Autoritäten und Gewissheiten und schreitet von dort zu dem einzig gewissen Fundament der Erkenntnis, dem sum cogitans, fort, um zur Befestigung einer unbezweifelbaren Erkenntnis, die über die bloße Selbstgewissheit des denkenden Subjekts hinausgeht, dann doch wieder auf Gott zurückzukommen, mit dessen Begriff er das Zutrauen in die Zulänglichkeit der menschlichen Vernunft zu befestigen vermag. Nicht anders Spinoza, der in seinem Denken aufgrund der Einsicht in den unüberwindbaren Partikularismus eines rein subjektiven Ausgangspunkts von Vornherein beim Begriff Gottes als der alles umfassenden Substanz einsetzt, die sowohl das Denken als auch das Sein umfasst und daher zum Garanten der Wahrheitserkenntnis werden kann. Der Gott Spinozas ist ein philosophischer Gott, der in scharfem Gegensatz zum Gott Abrahams, Isaaks und Jakobs steht. Seine die Gottes-, Selbst- und Naturerkenntnis ermöglichende Metaphysik muss insofern als eine Anti-Theologie angesprochen werden (Walther 1971).

Neben den Bestrebungen zur Abgrenzung und Überbietung von Religion in der politischen Praxis, in der politischen Theorie, in der Epistemologie und Metaphysik finden sich ähnliche Tendenzen auch im Naturrecht, in der Moralphilosophie und in den Naturwissenschaften. Man kann in diesem Zusammenhang an Hugo Grotius denken, der einräumte, dass sich das Naturrecht so formulieren ließe, als ob es Gott nicht geben würde, oder an Pierre Bayle, der den traditionellen Zusammenhang von Moral und christlichen Glauben auflöste und behauptete, dass auch Atheisten moralisch handeln könnten. Dabei zielten diese naturrechtlichen und moralphilosophischen Fundierungsansätze nicht darauf ab, den Glauben an die Existenz und das Wirken Gottes auszuschließen, sondern nahmen zur Befestigung und Begründung ihrer Einsichten ganz im Gegenteil das christliche Gottesbild ihrer Zeit gerade in Anspruch.

Der Überwindung der konfessionell geschlossenen Strukturen durch außerkirchliche Kräfte entsprach der sich gleichzeitig vollziehende Aufbruch im Inneren des Christentums durch Puritanismus, Pietismus, Jansenismus sowie durch Deismus, Spiritualismus und Mystik. Diese innerchristlichen Bestrebungen des nachkonfessionellen Zeitalters zeichneten sich erstens die Kritik an einem veräußerlichten Christentum, an Dogmatismus, Ritualismus und Wunderglaube, zweitens die Überwindung eines verengten Konfessionalismus, drittens die Verinnerlichung der Glaubensvollzüge, viertens die Abwehr päpstlicher Macht- und Herrschaftsansprüche sowie fünftens das Bemühen um einen Ausgleich von Glaube und Vernunft. Im Hintergrund dieser Bestrebungen stand der Versuch, dem Christentum von innen heraus eine neue allgemeingültige Grundlage zu geben, sei es eine neue Innerlichkeit, eine neue Einheit mit Gott oder ein neuer vernunftgemäßer Glaube, um den entstandenen Widerstreit der Bekenntnisse auf dieser Grundlage zu überwinden.

als ein großes Forum des Wettbewerbs zwischen um Geltung ringenden geistigen, politischen und sozialen Bestrebungen bezeichnen. Nach der oben vorgeschlagenen Bestimmung von Merkmalen der Moderne stellen Wettbewerbsforen Antriebsmotoren der gesellschaftlichen Dynamisierung dar. 
Im Laufe des 17. Jahrhunderts traten innerhalb und außerhalb des Christentums die unterschiedlichsten Bestrebungen auseinander: lutherische Dogmatik und individueller Spiritualismus, philosophische Spekulation und naturwissenschaftlichmathematischer Empirismus, päpstlicher Zentralismus und politische Souveränität, konfessionelle Selbstbehauptung und naturrechtlich begründete Aussöhnung. Ob die Cartesianer den archimedischen Punkt in der Selbstgewissheit des erkennenden Subjektes anvisierten oder Spinoza alle Erkenntnis mit mathematischer Notwendigkeit aus der sich selbst begreifenden Substanz abzuleiten versuchte, ob die spiritualistischen Sekten alle äußeren Rituale verwarfen und allein auf das innere Licht der Seele vertrauten, ob die Politiker in der Nachfolge Hobbes die fürstliche Herrschaft mit einer unantastbaren Souveränität ausstatteten oder Aufklärer nach einer universalen vernünftigen Moral Ausschau hielten - immer ging es darum, ein allgemeingültiges Fundament aufzurichten und an die Stelle der einst als unbezweifelbar erachteten religiösen Wahrheit eine neue unbezweifelbare Wahrheit zu setzen. Vermochte die Metaphysik die Grundlage der neuen Zeit abzugeben oder sollte es eine kirchenkritische Mystik sein oder eine religiös unabhängige Moral? Oder bot vielleicht der Deismus mit seinen Bemühungen um eine Harmonisierung zwischen dem Wunderglauben der Offenbarung und den Rationalitätsanforderungen der Vernunft einen Ausweg? Oder die Besinnung auf das tief in die Herzen der Menschen eingeschriebene natürliche Recht?

Man polemisierte gegen den Aberglauben, den Glauben an Wunder, gegen Vorurteile, den Common Sense, gegen den Zwang der Gewissen, das römische Joch, ganz allgemein gegen Autoritäten, gegen die christliche Überlieferung, die Göttlichkeit der Bibel. Wie ließen sich diese divergierenden Kräfte noch bändigen (Hazard 1939, S. 119)? Waren die neuen Grundsätze der Moral, der Metaphysik, der Vernunft, des Naturrechts tatsächlich überzeugend? Oder war es nicht besser, zum alten Glauben zurückzukehren und Europa auf diese Weise zu einigen, so wie es Ludwig XIV. mit seiner Rekatholisierungspolitik anstrebte? Aber war es nicht gerade dieser mit politischen Mitteln durchgesetzte Letztgültigkeitsanspruch, dem man den Prozess machte? Leibniz suchte die Uneinigkeit Europas durch die Aussöhnung von Protestantismus und Katholizismus zu überwinden. In seinen Briefen an Bossuet bekannte er sich zum Prinzip der Katholizität und schlug die Einrichtung eines ökumenischen Konzils vor, das in einem offenen Prozess Kompromisse aushandelt, auf die sich beide Seiten einlassen können, und in dem wechselweise die Protestanten den katholischen und die Katholiken den protestantischen Standpunkt einnehmen und sich so einander annähern. Bossuet jedoch, der die Protestanten durchaus zurückgewinnen wollte, erklärte, wenn Leibniz das Katholizitätsprinzip anerkenne, dann möge er sich doch zum Katholizismus bekehren. Die Protestanten könnten nicht mit einer praktischen Versöhnung beginnen, um dann eine Versammlung einzuberufen, die sich gütlich über die zu akzeptierende Doktrin verständigt; wenn sie in die Gemeinschaft der römischen Kirche zurückkehren wollten, müssten sie sich zuerst unterwerfen. Welten stießen hier aufeinander. Der eine wollte verhandeln, Kompromisse finden und durch tentative Übernahme des fremden Standpunkts ausloten, bis wohin man einander entgegenkommen kann; der andere erwartete, das Gegenüber möge überlaufen und die Gültigkeit der einen unwandelbaren Wahrheit anerkennen. Die Bereitschaft zur Selbstrelativierung stand gegen den Anspruch auf Gehorsam, 
der Geist der Toleranz und Offenheit gegen die Behauptung unbezweifelbarer Autorität.

\subsection{Der Durchbruch zur europäischen Moderne: Die Zähmung der Religion und die Akzeptanz der Pluralität von Geltungsansprüchen}

In diesem Gegensatz freilich spiegelt sich bereits der Anbruch einer neuen Zeit. Die Vorstellung von einer ,kirchlichen Gesamtkultur“ (Troeltsch 1911, S. 27) konnte nicht länger aufrechterhalten werden. Nicht nur das Reich war religiös plural geworden. Aufgrund des Konfessionswechsels der Landesherren, etwa in Brandenburg und Sachsen, aufgrund des Rechts der Bevölkerung, den Konfessionsstand des Normaljahres von 1624 zu behalten, aufgrund von Mobilität und mannigfachen Ausnahmeregelungen, etwa für die Reichsstädte, wurde das Recht der Landesherren, über die Konfession der Untertanen zu bestimmen, entscheidend eingeschränkt und erhöhte sich der Grad der religiösen Pluralität auch innerhalb der Territorien. Das lange verfolgte Ziel einer Vergleichung der Konfessionen ließ sich nicht umsetzen, und damit rückte auch das Ideal der Einheit von Religion und Politik außer Reichweite. Die Erwartung, sich auf die eine religiöse Wahrheit einigen zu können, obschon im Westfälischen Frieden formal noch einmal bekräftigt, war Mitte des 17. Jahrhunderts bereits weitgehend zerrüttet (Reuter 2014, S. 80).

Gleichwohl trieb die Hoffnung, der Gesellschaft ein neues Fundament geben zu können, viele der politischen, rechtlichen, philosophischen und moralischen Unternehmungen im nachkonfessionellen Zeitalter noch an. Unübersehbar lebte in diesen Bestrebungen der Geist des untergegangenen Systems fort. Insofern trugen diese Bestrebungen noch Züge dessen, wovon sie sich abgrenzten. Im Übergang zum und im Laufe des 18. Jahrhunderts gewann jedoch immer mehr die Überzeugung die Oberhand, dass die Suche nach einer Verankerung des Wahren, Guten und Schönen in der Transzendenz nicht zum Ziel führen wird, dass das, was gelten soll, sich nicht aus dem Absoluten ableiten lässt, sondern in einem offenen Prozess ermittelt und ausgehandelt werden muss, dass Geltung empirisch und intersubjektiv herzustellen ist und es in der Bestimmung des Wahren, Guten und Schönen Grenzen des Konsenses gibt.

Es entspricht diesem neuen Geist, dass die Aufhebung des Ediktes von Nantes unter den intellektuellen Zeitgenossen in Europa allgemein Empörung auslöste. Der Versuch, unter Anwendung von physischer Gewalt Gewissenszwang auszuüben, widersprach diametral den Tendenzen des Zeitalters, die auf Autoritätskritik, auf Abwehr vernunftwidriger Dogmen, auf Überwindung der konfessionellen Spaltung, auf religiöse Verinnerlichung und auf Ausweitung individueller Autonomie gestimmt waren. Die Aufhebung des Edikts von Nantes markiert so das Ende des Siegeszugs der Gegenreformation (Hazard 1939, S. 111). Ging es im 17. Jahrhundert und teilweise bis zu Beginn des 18. Jahrhunderts noch darum, welche geistige, religiöse und politische Bewegung die universellen Geltungs- und Superioritätsansprüche der römischen Kirche zu beerben vermag, und wurden bis dahin die einzelnen Positionen noch mit geradezu religiöser Leidenschaft vertreten, so kam es im Laufe des 18. Jahrhunderts mehr und mehr zum Rückbau der religiösen, philosophischen und moralischen Ausschließlichkeitsansprüche und zur Domestizierung der religi- 
ös aufgeladenen Letztgültigkeitsbehauptungen. An die Stelle einer um jeden Preis ausgefochtenen Konkurrenz trat mehr und mehr das Bemühen um die Begrenzung aufgerichteter Geltungsansprüche.

$\mathrm{Zu}$ tun hat diese Zurücknahme absoluter Wahrheits- und Überlegenheitsansprüche vor allem mit den Folgen des Zusammenbruchs der kirchlichen Einheit des westlichen Christentums. Aufgrund der konfessionellen Pluralisierung vollzog sich im Habsburger Reich, wie bereits dargestellt, auf der gesamtgesellschaftlichen Ebene eine Differenzierung zwischen Religion und Recht, mit deren Hilfe die religiöse Neutralität des Rechts sowie die paritätische Behandlung der Konfessionen garantiert werden konnten. Dabei bedeutete die Überordnung des Rechtsfriedens über die Kirchenparteien und die reichsrechtliche Parität der Konfessionen eine Relativierung der konfessionellen Absolutheitsansprüche, von der langfristig ein beachtlicher Säkularisierungsschub ausging (Heckel 2007, S. 28).

Aber auch auf der Ebene der Territorien hatte die religiöse Pluralisierung relativierende und säkularisierende Effekte. Auf der einen Seite kam der weltlichen Obrigkeit formell noch immer das Aufsichtsrecht über die Verkündigung des wahren Evangeliums zu. Auf der anderen musste sie jedoch zugleich für die Sicherung des sozialen Friedens sorgen - eine Aufgabe, der sie sich stellte - und zwar nicht nur im Reich, sondern auch etwa in England oder in den Niederlanden -, indem sie die Mehrheitsreligion gegenüber den Minderheitsreligionen bevorzugte und die Religionsausübung der letzteren unsichtbar hielt und in den Privatraum abdrängte. Eine derartige Ungleichbehandlung gefährdete jedoch genau das, was durch sie erreicht werden sollte: den sozialen Frieden, so dass die Herrscher im gleichen Zug gezwungen waren, den benachteiligten religiösen Gruppen, deren politische Loyalität und deren ökonomische Arbeitskraft sie nicht verlieren wollten, wiederum mit Privilegien und besonderen Rechten auszustatten. Zur Sicherung des friedlichen Zusammenlebens mussten sie mithin zwischen Untertanengehorsam und Glaubenstreue, zwischen politischer Loyalität und religiöser Wahrheit unterscheiden und darauf verzichten, die politische Beurteilung ihrer Untertanen von deren Zustimmung zur Mehrheitskonfession abhängig zu machen (Stollberg-Rilinger 2019, S. 6). Das bedeutet, dass sie nicht nur eine Differenzierung zwischen politischer und religiöser Ordnung vornahmen, sondern auch politische Maßstäbe religiösen vorordneten. Die Staatszwecklehre änderte sich. Nicht länger kam dem Staat vor allem die Aufgabe $\mathrm{zu}$, als Beauftragter Gottes die Verkündigung des Evangeliums zu sichern und für die Wahrheit einzutreten, sondern vor allem die, ,das Wohl, den Nutzen des Staates, die salus publica“ zu fördern (Sehling 1914, S. 35). Der Staat habe sein Ziel in sich selbst: im Erhalt seiner Souveränität und in der Sicherung der öffentlichen Ordnung; die Religion könne durch ihren Beitrag zur Moral und zum gesellschaftlichen Zusammenhalt der Staatsräson allenfalls dienen (Schlaich 1969, S. 78 f.). Wie schon mit der paritätischen Behandlung der Konfessionen auf Reichsebene ging also auch mit der Überordnung der an der Sicherung des sozialen Friedens orientierten Staatsräson über die herrschaftliche Verpflichtung zur Durchsetzung der religiösen Wahrheit eine Relativierung religiöser Geltungsansprüche einher.

Dabei war die Unterordnung der Kirche unter die Politik in die langfristigen politischen Bestrebungen zur Stärkung der Staatsgewalt eingebettet. Die Kompetenz der Kirche wurde auf die inneren geistlichen Angelegenheiten beschränkt, während die 
Regelung der äußeren Dinge (ius circa sacra) mehr und mehr in die Verfügungsgewalt des Staates überging. Waren im 16. und 17. Jahrhundert und teilweise bis weit ins 18. Jahrhundert hinein politische Herrschaft und Kirche noch eng verflochten und konnte die Kirche in dieser Zeit, über den langen Arm der Staatsgewalt noch einen unmittelbaren Einfluss auf alle Bereiche des Lebens ausüben und nicht nur den Staat, sondern auch das Rechtswesen, das Bildungswesen, die Armenfürsorge, Schule und Universitäten durchdringen, so lockerte sich im Laufe des 18. Jahrhunderts und insbesondere in dessen letztem Drittel der politisch vermittelte Zugriff des Christentums auf die Gesellschaft. Trotz staatlicher Kirchenhoheit unterstützte der Staat die Kirche nicht mehr bei der Umsetzung kirchlich verhängter Strafen. Damit war die Kirchenzucht faktisch abgeschafft. Die Privatbeichte wurde durch die allgemeine Beichte ersetzt, die Katechismusprüfungen fanden nicht mehr in der Öffentlichkeit statt, der Druck auf den Besuch des Gottesdienstes und des Abendmahls ließ nach. Es kam zu einer zunehmenden Entflechtung von politischer Herrschaft und Konfessionschristentum, zu einer Art Entriegelung der Interessenallianz zwischen Kirche und Staat, die das soziale Leben einst umfassend bestimmt hatte, und damit zu einer bislang nicht gekannten Freisetzung und Entfaltung sozialer Kräfte. Die Folgen waren mannigfach.

Am wichtigsten in diesen teilweise unverbundenen, teilweise parallel laufenden, teilweise miteinander verknüpften Aufbrüchen war die Herausbildung einer neuen Freizeit- und Geselligkeitskultur, die viele Schichten einbezog und nicht selten in direkte Konkurrenz zum Gottesdienst als dem bisherigen Zentrum der gesellschaftlichen Kommunikation trat. Bildete der Stand bislang den größtmöglichen Lebensrahmen eines jeden Einzelnen und blieb angesichts dessen jede Vorstellung von einer Gesamtgesellschaft unterentwickelt (Osterhammel 2016, S. 653), so entstand mit den neuen Kommunikationsformen so etwas wie Gesellschaft, ein öffentlicher Raum der Diskussion und des sozialen Austauschs. Es waren Kaufleute, Bankiers und Geschäftsleute, finanzkräftige Bürgersleute und Unternehmer, Schriftsteller, Bibliothekare und Philosophen, aber auch Teile des Adels, die sich daranmachten, in den Städten und teilweise auch an den Höfen eine neue Diskussionskultur zu errichten (Hölscher 1990, S. 603, 2005, S. 95-100). Man traf sich in Kaffeehäusern, Clubs, an der Börse, in Teegesellschaften, Salons und literarischen Zirkeln und debattierte über den moralischen Zustand der Gesellschaft, über philosophische Neuerscheinungen und neueste Ereignisse am Hof. Wissenschaftliche Akademien wurden gegründet, ebenso Geheimbünde und Freimaurerlogen. Das Leseverhalten transformierte sich von der intensiven Lektüre einiger weniger, vor allem religiöser Schriften zur extensiven Lektüre einer Vielzahl von literarischen Werken. ${ }^{15}$

Die Entstehung dieses neuen Markts der Ideen, der Unterhaltung und der Lektüre ging Hand in Hand mit einer weiteren Relativierung des Geltungsanspruchs moralischer, philosophischer und religiöser Lehren. Die Abhängigkeit der Wahrheit von der Perspektive des Publikums kam in den Blick. Von der Behauptung objektiv gültiger Wahrheiten wurde mehr und mehr umgestellt auf individuelle Erfahrung.

15 Die Entfaltung der bürgerlichen Emanzipationsbewegung im Schatten des Absolutismus und ihre Ausdehnung in den öffentlichen Raum hinein wird anschaulich beschrieben von Reinhart Koselleck (1973, S. 49-103). Vgl. auch Habermas (1990b). 
An die Stelle der Metaphysik und der Behauptung letztgültiger Gewissheit trat der Empirismus. John Locke - ein Vorläufer des neuen Geistes - erklärte, man solle die Metaphysik auf sich beruhen lassen und sich an das halten, was man sieht. Das menschliche Handeln, Denken und Wahrnehmen löste sich mehr und mehr von der Orientierung auf eine Zentralperspektive. Waren im 17. Jahrhundert die Denker der großen rationalen Systeme wie Descartes, Spinoza, Malebranche oder Leibniz noch durchgängig den Prinzipien der Metaphysik verpflichtet und hatte selbst ein kritischer Geist wie Descartes (1972 [1641], S. 60 (5, 18)) die Gewissheit und Wahrheit des Wissens noch allein durch die Erkenntnis des wahren Gottes als verbürgt angesehen, so ist im Sensualismus eines Hume - nur ein Jahrhundert nach Descartes - der metaphysisch begründete Zugang zur Wirklichkeit preisgegeben. In der Nachfolge John Lockes begreift Hume Wissen als eine durch wiederholte Erfahrung gewonnene, also als eine empirisch bedingte und empirisch auch wieder aufhebbare Form der Erkenntnis. Für Kant ist die empirische Erkenntnis an die im Subjekt liegenden Bedingungen der Möglichkeit synthetischer Erkenntnis a priori gebunden. Damit begründet er nicht nur die Autonomie des Wissens, sondern weist diesem auch seine Grenzen zu, denn eine solche Erkenntnis reicht nur bis zu den Gegenständen möglicher Erfahrung und muss nichtempirische Ideen wie Gott, Freiheit und Unsterblichkeit als Postulate aus ihrem Gegenstandsbereich ausscheiden. Kant schränkt Erkenntnis mithin auf den Umkreis methodisch gesicherten Wissens ein. Der moderne Naturwissenschaftler lasse sich zwar noch von der Natur belehren, aber nicht mehr wie ein Schüler, der von seinem Lehrmeister, von der Natur, alles vorgesagt bekommt, sondern wie ein „Richter, der die Zeugen nötigt, auf die Fragen zu antworten, die er ihnen vorlegt" (Kant 1956 [1787], S. 19). Nahm man bisher „an, alle unsere Erkenntnis müsse sich nach den Gegenständen richten“, so fragt Kant nunmehr, ,ob wir nicht [...] damit besser fortkommen, daß wir annehmen, die Gegenstände müssten sich nach unserer Erkenntnis richten“ (20). Nur unter dieser Voraussetzung könne ein methodologisch gesicherter Erkenntnisfortschritt gelingen.

Damit ist der Primat der Methode vor der Wahrheit begründet oder, wie wir auch sagen können, der Primat der Selbstreferentialität der Erkenntnis. Wenn die Wissenschaft die Wirklichkeit erkennen will, muss sie sich kritisch auf sich selbst beziehen und ihre eigenen Erkenntnisvoraussetzungen zum Thema machen. Ein ähnliches Begründungsverhältnis treffen wir auch in der politischen Theorie an. Hobbes hatte die Souveränität der Staatsmacht trotz der Umgründung der Rechtfertigung ihrer Legitimität von Gott auf das Volk noch unumschränkt gedacht und jeden Rechtfertigungsbedarf vor dem Volk ausgeschlossen; Locke und Montesquieu hingegen nehmen durch die Vorstellung von der Gewaltenteilung eine interne Selbstrelationierung der Staatsmacht vor und in Zusammenhang damit eine Orientierung der Politik an den Rechten und Bedürfnissen der Untertanen, an dem Willen des Volks. Mit der Umgründung von letzter Gewissheit auf selbstreferentiell vermittelten Umweltkontakt ist folglich auch eine Tendenz zur Selbstbegrenzung verbunden, zur internen Berücksichtigung externer Gesichtspunkte und zur Relativierung systemeigener Rationalität. Die die Moderne charakterisierende Steigerungsdynamik wird schon in der Aufklärung durch eine Tendenz zur reflexiven Selbstbeschränkung gebrochen. Diese Tendenz findet sich auch im aufklärerischen Verhältnis zur Religion, etwa in der Forderung der Aufklärung nach Toleranz. In der Ringparabel behandelt Lessing 
den Islam, das Judentum und das Christentum nicht nur als gleichwertig, er äuBert auch Skepsis gegenüber dem von ihnen erhobenen Anspruch auf überzeitliche Wahrheit. „Der echte Ring“, so lässt er Nathan sagen, ,vermutlich ging verloren“. Wenn überhaupt, so lasse sich die Wahrheit einer Religion am sittlichen Handeln ihrer Anhänger erkennen, denn der wahre Ring besitze die Fähigkeit, ,beliebt zu machen, vor Gott und Menschen angenehm“ (Lessing 1954 [1778], S. 407) - womit ein weiteres Mal das kritische Urteil des aufgeklärten Publikums ins Spiel kommt.

Hinter der ethischen Forderung, die Lessing mit seiner Ringparabel formulierte, stand ein radikaler Wandel des Weltbildes - und damit ist ein weiterer Säkularisierungsschub angesprochen, der mit den Aufbrüchen des Zeitalters eng zusammenhing. Im 16. und 17. Jahrhundert herrschte weithin ein Gottes- und Menschenbild vor, das den Menschen als ein durch die Erbsünde verdorbenes Wesen darstellte, dem allein die Gnade Gottes aus seinem erbärmlichen Zustand heraushelfen kann. Nur wer zu Gott umkehre, könne auf die Gnade Gottes hoffen; dem nicht reuigen Sünder aber drohe der ewige Tod in der Hölle. Dabei sei Umkehr umso mehr geboten, als das Ende der Welt - dieses irdischen Jammertales - kurz bevorstehe (Kittsteiner 1995, S. $121 \mathrm{ff}$.). Hundertfünfzig Jahre später hatte sich das Blatt gewendet. Aus dem Gott, der entweder zum ewigen Unheil verdammte oder aber nur unverdiente Gnade walten lassen konnte, war ein liebender Gott geworden, dessen gütigem Wesen es widersprechen würde, für zeitliche Sünden ewige Höllenstrafen vorzusehen. Der Mensch, erklärte Joachim Spalding (2002 [1772], S. 193f.), das Haupt der neologischen Theologie, sei nicht von allem Anfang an zur Sünde und damit zur ewigen Höllenqual bestimmt; seine schöpfungsmäßige Bestimmung liege vielmehr in seiner Unsterblichkeit (Spalding 2006 [1748]). Die Erlösung des Menschen vollziehe sich in Form eines beständigen Hinaufsteigens bis hin zur himmlischen Vollkommenheit (Beutel 2014, S. 18). Der Mensch könne nicht nur zwischen Gut und Böse unterscheiden, sondern das Gute auch tun. Tugendhaftes Handeln erhöhe sein Anrecht auf Teilhabe am Reich Gottes. Jeder Einzelne müsse die Bahn, auf der das Menschengeschlecht zu seiner Vollkommenheit voranschreite, durchlaufen, der eine früher, der andere später, heißt es in der „Erziehung des Menschengeschlechts“, in dem Lessing (1956 [1780]: § 92 und § 98, S. 614f.) für den Fall, dass der Einzelne den ihm aufgetragenen Weg nicht in einem einzigen Leben vollenden könne, auf den Gedanken einer wiederholten Rückkehr des Menschen auf Erden zurückgreift, in der der Mensch so viele Kenntnisse und Fertigkeiten anhäufen könne, wie zu seiner im Unendlichen liegenden Vervollkommnung erforderlich sei. Das Menschenbild hat sich aufgehellt und mit ihm das Bild von Gott und von der Zukunft der Welt. Der Mensch ist nicht länger ein der Gnade bedürftiger Sünder. Nein, er kann die Welt gestalten, sich selbst durch tugendhafte Lebensführung verbessern und kommt in seinem Vervollkommnungsstreben niemals an ein Ende. Zur Erfassung dieses Umbruchs vom pessimistischen zum optimistischen Menschen- und Weltbild spricht Koselleck (1989, S. 320 f.) von der Verzeitlichung von Perfektionsidealen.

Für die semantischen Veränderungen, die sich in der Zeit vom konfessionellen Zeitalter bis zur Spätaufklärung vollzogen, dürften wirtschaftliche, technische und politische Wandlungsprozesse eine gewichtige Rolle gespielt haben. Unter Zurückdrängung ständischer Privilegien, kirchlicher Herrschaftsrechte und geistlicher Immunitäten versuchte die staatliche Gewalt, umfassende Reform- und Modernisie- 
rungsmaßnahmen durchzuführen, eine einheitliche Finanzpolitik durchzusetzen und eine unabhängige Bürokratie zu installieren (Stollberg-Rilinger 2006, S. $194 \mathrm{ff}$.). Nicht Rückkehr zum guten alten Herkommen strebte die Staatsmacht in von aufgeklärten Monarchen beherrschten Ländern wie Österreich, Russland oder Preußen an. Vielmehr ging es darum, unter Eingriff in die städtischen Zunftordnungen die marktwirtschaftliche Konkurrenz zu fördern, mit Hilfe von Rationalisierungsmaßnahmen die Effizienz der stadt- und landwirtschaftlichen Produktion zu erhöhen, die verkehrstechnische Infrastruktur auszubauen, das Rechtssystem zu vereinheitlichen, kirchliche Feiertage abzuschaffen, die Schulen zu kontrollieren, die medizinische Versorgung zu verbessern, das Landvolk aufzuklären, also darum, die Wirtschaftskraft der Untertanen zu steigern, um so aus ihnen gute Steuerzahler zu machen. Aufgrund verbesserter landwirtschaftlicher Anbaumethoden, aufgrund der Erhöhung der landwirtschaftlichen Nutzfläche, der Einführung neuer Kulturpflanzen sowie der Entwicklung der öffentlichen und privaten Hygiene im 18. Jahrhundert stieg die Lebenserwartung signifikant an (Wrigley 1969; Marschalck 1984). Mit der Erweiterung der technologischen und wirtschaftlichen Gestaltungskapazitäten nahm aber auch das Zutrauen des Menschen in seine eigenen Handlungsmöglichkeiten zu.

Wenn nicht alles täuscht, vollzog sich in der Gleichzeitigkeit dieser in vielerlei Hinsicht miteinander zusammenhängenden politischen, kulturellen, wirtschaftlichen, sozialen, literarischen und philosophischen Aufbrüche der Durchbruch zur Moderne. Ausgelöst durch die konfessionelle Pluralisierung der religiösen Wahrheit bildeten sich unter Verzicht auf eine im Absoluten verankerten Zentralperspektive miteinander ringende, auf Autonomie insistierende Praktiken und Diskurse heraus, die teilweise eng miteinander verschränkt waren, teilweise einen selbstorganisatorischen Charakter trugen, sich teilweise aber auch durch Konflikt und Konkurrenz wechselseitig stimulierten, ${ }^{16}$ jedenfalls nicht mehr kirchlich oder theologisch kontrolliert werden konnten. Dabei zeichneten sich diese Praktiken und Diskurse zunächst einmal durch eine Tendenz zur Abstandnahme gegenüber Religion und Kirche aus. 1791 wurde die Trennung von Kirche und Staat in den USA in der Verfassung verankert, 1795 in Frankreich, 1796 in den Niederlanden. Im Allgemeinen Landrecht für Preußen von 1794 wurden die Kirchen, die einst mit dem Anspruch aufgetreten waren, der wahren Lehre gesamtgesellschaftlich zum Sieg zu verhelfen, zur rechtlichen Organisationsform eines Vereins oder einer ,geistlichen Gesellschaft“ herabgestuft (Hattenhauer 1970, S. 542-584). Zugleich setzte sich um 1800 auch die Idee durch, Bildung und Erziehung zu eigenständigen, von Herkunft und Stand, aber auch von Tradition und Autorität unabhängigen Zielen zu erklären, bestimmt nicht mehr durch Geburt, sondern durch Talent und Leistung (Nipperdey 1983, S. 60). Die kirchliche Verfügung über die Schule wurde gelockert, und der Staat übernahm mit der Einführung des Allgemeinen Landrechts in Preußen die Kontrolle über die Erziehung (ebd., S. 56). An der Wende vom 18. zum 19. Jahrhundert gewannen die Universitäten an Unabhängigkeit; die konfessionell geprägten Universitäten wurden mehr und mehr durch staatlich getragene Universitäten ersetzt (ebd., S. 65).

\footnotetext{
16 Die Wirksamkeit der damit angedeuteten Mechanismen des Konflikts, der Transformation, der Selbstorganisation sowie der Konkurrenz und der Verflechtung bedürfen weiterer Ausarbeitung.
} 
Diese Autonomisierungstendenzen der Politik, Erziehung und Bildung spiegelten sich auch auf der semantischen Ebene und zwar nicht nur, wie bereits dargestellt, in der Epistemologie und der politischen Theorie, sondern auch in der Ästhetik, der Moralphilosophie und nicht zuletzt auch die Religionstheorie selbst. Betrachtete etwa Shaftesbury Anfang des 18. Jahrhunderts die Vollkommenheit Gottes noch als die Quelle alles Schönen und lag es daher für ihn nahe, das Schöne und das Gute als „ein und dasselbe“ anzusehen (Shaftesbury 1981, S. 176, 246, 324, 346), so unterscheidet Winckelmann in der zweiten Hälfte des Jahrhunderts zwischen Schönheit, Sittlichkeit und Wahrheit. In seinen Augen drückt sich das Schöne im Kunstwerk als etwas in sich selbst Sinnvolles und Bedeutsames aus, obschon er es noch immer als einen Abglanz der höchsten Schönheit, die in Gott ist, fasst (Kühne-Bertram 1992, S. 1374). Die Herauslösung der Ästhetik aus der Metaphysik, verbunden mit der Akzeptanz des Schönen als eines eigenständigen Geltungsbereichs, wird dann wiederum von Kant vollzogen. Das Schöne sei schön um seiner selbst willen, durch die Abwesenheit von mit ihm verfolgten Interessen, und dadurch, dass es als allgemein und notwendig, also als nicht kontingent empfunden werde (Kant $1900 \mathrm{ff}$. [1790]: $\S 5$ (B 16), § 17 (B 61), §§ 18. 22 (B 62, 68), 211, 236, 240).

Außerdem wird auch die Begründung der Moral als autonomer Lebensbereich und die Abkopplung ihrer Geltung von den Wahrheitsansprüchen der Religion durch Kant vollzogen. Der Mensch als freies Wesen bedürfe keiner ,Idee eines anderen Wesens über (sich), um seine Pflicht zu erkennen“ (Kant 1968 [1793], S. 3). Was gut und böse sei, könne er selbst beurteilen. Die an die Priestervorschriften sich haltenden Religionspraktiken und der gehorsame Glaube an das Wort hingegen verletzten das menschliche Gewissen, denn die einzige Maxime des Gewissens sei, ,nichts zu glauben als das, was von der eigenen Vernunft geprüft werden kann“ (Kittsteiner 1995, S. 269, mit Bezug auf Kant (1968) [1793], S. 188f.).

In der Nachfolge Kants verschaffte Friedrich Daniel Ernst Schleiermacher (1999 [1799]) schließlich auch der Religion ein autonomes Fundament. Neben Moral und Metaphysik als autonome Fakultäten des menschlichen Handelns und Wissens stellte Schleiermacher die Religion, die im Gefühl ihre weder moralisch noch metaphysisch auflösbare Basis besitzt. Im „Bewusstsein der schlechthinnigen Abhängigkeit“, in dem der späte Schleiermacher (1984 [1830/31], S. 3-6) die unableitbare Eigenständigkeit der Religion begründet sah, realisiere der Mensch die emotionale Anerkenntnis einer empirisch nicht einholbaren transzendentalen Voraussetzung all seines Denkens, Tuns und Wahrnehmens.

Einen systematischen Ausdruck der im ausgehenden 18. Jahrhundert sich vollziehenden strukturellen und semantischen Differenzierungsprozesse findet sich in Schillers Theorie des modernen Zeitalters, wie er sie in seinen Briefen „Über die ästhetische Erziehung des Menschen“ von 1795 niederlegte. In dieser Abhandlung würdigt Schiller die Fortschritte, die die Gesellschaft auf dem Gebiet der Technik, der Wissenschaft und des Handwerks infolge der Arbeitsteilung und Spezialisierung gemacht hat. Diese Erfolge hätten zwar zu einer Verarmung der im Einzelnen angelegten Kräfte geführt und diesen zu einem bloßen „Abdruck seines Geschäfts“ gemacht (Schiller 1993 [1795], S. 584). Die Kritik an der Fragmentierung und Verzwecklichung des Individuums führt Schiller aber keineswegs dazu, die Rücknahme des gesellschaftlichen Fortschritts zu fordern und die Verwirklichung einer gesell- 
schaftlichen oder individuellen Ganzheit gegen die arbeitsteilige Spezialisierung der Gesellschaft einzuklagen. Vielmehr begreift er die gesellschaftliche Komplexitätssteigerung und die Zersplitterung des menschlichen Wesens als zusammengehörig. Für ihn ist klar, „dass, so wenig es auch den Individuen bei dieser Zerstückelung ihres Wesens wohl werden kann, doch die Gattung auf keine andere Art hätte Fortschritte machen können“ (Schiller 1993 [1795], S. 586). Wissenschaft und Kunst können sich nur von innen her entfalten. „Der politische Gesetzgeber kann ihr Gebiet sperren, aber darin herrschen kann er nicht." (Schiller 1993 [1795], S. 593) Die Eigenlogik von Funktionssystemen von außen zu beeinflussen, kann nur destruktive, aber keine instruktiven Effekte haben, so fasst Luhmann (1997, S. 753) unter ausdrücklichem Bezug auf Schiller den Sachverhalt.

\section{Fazit}

Am Ende des 18. Jahrhunderts können wir die Umrisse einer sich neu herausbildenden Gesellschaftsformation erkennen: den Vorschein einer funktional differenzierten Gesellschaft. Sowohl auf der sozialstrukturellen Ebene als auch auf der Ebene des Diskurses sind die Prozesse einer Differenzierung unterschiedlicher Wert- und Gesellschaftssphären weit vorangeschritten. Ging die römische Kirche im Hochmittelalter in der Behauptung einer sozial unableitbaren theologischen Eigenrationalität und einer damit verbundenen Institutionalisierung der gesellschaftlichen Entwicklung voran, so war es ihr auf theologischer Grundlage aufgerichteter Superioritätsanspruch über alle anderen gesellschaftlichen Bereiche, der diese dazu herausforderte, sich gegen diese Zumutung zu behaupten und ihrerseits Eigenrationalitäten auszubilden. Eine Vielzahl von Faktoren spielte in die Ausbildung dieser Selbstbehauptungstendenzen hinein, wobei die relative Schwäche der politischen Zentralgewalt sowohl auf die Radikalisierung theologischer Gültigkeitsansprüche als auch auf die Entfaltung der Gegenkräfte stark begünstigend wirkte. Nach dem Zusammenbruch der kirchlichen Einheitskultur in der Reformation, nach den vergeblichen Versuchen, die eine religiöse Wahrheit auf konfessioneller Basis wiederherzustellen, und nach dem Scheitern der Bestrebungen, eine neue in der Transzendenz verankerte Zentralperspektive zu etablieren, gewannen Tendenzen der gesellschaftlichen Ausdifferenzierung die Oberhand, die sich teilweise in scharfer Abgrenzung von der Kirche, insbesondere der römischen Kirche, teilweise auch unter Aufnahme religiöser Traditionen und in dem Bemühen um ihre Harmonisierung mit den ausdifferenzierten Perspektiven, teilweise aber auch losgelöst von religiösen Geltungsansprüchen entwickelten. Verbunden waren diese Differenzierungsprozesse mit Tendenzen der Individualisierung und der gleichzeitigen Institutionalisierung, also mit Tendenzen der Differenzierung gesellschaftlicher Konstitutionsebenen, mit einer zunehmenden Konkurrenz zwischen den gesellschaftlichen Bereichen und einer daraus resultierenden Beschleunigung gesellschaftlichen Wandels. Wie wir sehen konnten, ließen sich auch bereits Anstrengungen einer rational begründeten Selbstbegrenzung erkennen. Damit waren die Weichen für die Emergenz der europäischen Moderne gestellt, die im 19. Jahrhundert zu einer umfassenden Verwandlung der Welt führen sollte. 
Ein zentraler kausaler Mechanismus für die Herausbildung einer polyzentrischen Gesellschaftsformation bestand, wie die vorangegangen Analysen gezeigt haben, in der Wirksamkeit konfligierender Geltungsansprüche. Das gilt bereits für die Zeit des Hochmittelalters, als der Herrschafts- und Weltdeutungsanspruch der Papstkirche alle nichtkirchlichen Bereiche dazu herausforderte, ihre eigenen Selbstbehauptungsstrategien zu verfolgen, entweder in der Form des offenen Kampfes oder in der Form einer Vermeidung des offenen Konflikts. Konfliktmechanismen trieben aber auch die Entwicklung im konfessionellen und nachkonfessionellen Zeitalter an, als sich die divergierenden Kräfte kaum noch bändigen ließen. Erst durch den Verzicht auf eine im Absoluten verankerte Zentralperspektive konnte der Konflikt minimiert werden und wurde es möglich, Überbietungsansprüche in Selbstbegrenzungstendenzen zu überführen und Zonen der Indifferenz zuzulassen.

Inwieweit diese Prozesse der Modernisierung endogen erzeugt, inwieweit sie exogen angestoßen oder sogar bestimmt waren, kann im hier gesetzten Rahmen nicht mehr erörtert werden. ${ }^{17}$ Die interkulturellen Verflechtungszusammenhänge in der Herausbildung der modernen Welt müssen weiteren Studien vorbehalten bleiben. Dabei wird es erforderlich sein, in diesen Analysen besonderen Wert auf die Beachtung der religiös-theologisch erzeugten Eigendynamik, auf die Schärfe der Absetzung des Religiösen vom Säkularen, also auf die Vorreiterrolle der Religion, sowie auf den begünstigenden oder inhibierenden Einfluss der politischen Zentralmacht zu legen.

Funding Open Access funding provided by Projekt DEAL.

Open Access Dieser Artikel wird unter der Creative Commons Namensnennung 4.0 International Lizenz veröffentlicht, welche die Nutzung, Vervielfältigung, Bearbeitung, Verbreitung und Wiedergabe in jeglichem Medium und Format erlaubt, sofern Sie den/die ursprünglichen Autor(en) und die Quelle ordnungsgemäß nennen, einen Link zur Creative Commons Lizenz beifügen und angeben, ob Änderungen vorgenommen wurden.

Die in diesem Artikel enthaltenen Bilder und sonstiges Drittmaterial unterliegen ebenfalls der genannten Creative Commons Lizenz, sofern sich aus der Abbildungslegende nichts anderes ergibt. Sofern das betreffende Material nicht unter der genannten Creative Commons Lizenz steht und die betreffende Handlung

$17 \mathrm{Zu}$ dieser Thematik liegt eine Fülle an Literatur vor. Diese von den Ansätzen der Globalgeschichte, der shared histories, der entangled modernities, der Kolonialgeschichte und der postcolonial studies beeinflusste Literatur geht davon aus, dass die Entstehung der europäischen Moderne nicht primär endogen verstanden werden kann, sondern aus ihrer Verflechtung mit außereuropäischen Regionen und mit der Kolonialgeschichte analysiert werden muss. (Vgl. anstelle vieler nur: Chakrabarty 2000; Conrad und Randeria 2002; Conrad 2013; Epple 2012). Wie bereits in der Einleitung zu diesem Aufsatz klargestellt, ist die Auseinandersetzung mit dem Gewicht endogener und exogener Faktoren, die die Emergenz der europäischen Moderne beeinflusst haben, nicht das Thema dieses Aufsatzes. Dass die Verhältnisbestimmung endogener und exogener Faktoren alles andere als einfach ist, macht aber bereits das Zitat eines der führenden Vertreter der Globalisierungsgeschichte deutlich. In seiner Geschichte des langen 19. Jahrhunderts schreibt Jürgen Osterhammel, es wäre ein ,Ausdruck kapriziöser Willkür, eine Geschichte ausgerechnet des 19. Jahrhunderts zu entwerfen, die von der Zentralität Europas absähe. Kein anderes Jahrhundert war in einem auch nur annähernden Maße eine Epoche Europas. [...] Nie zuvor hatte die westliche Halbinsel Eurasiens derart große Teile des Globus beherrscht und ausgebeutet [...]. Niemals wurde auch die europäische Kultur - weit jenseits der Sphäre kolonialen Zugriffs - dermaßen begierig aufgenommen. Das 19. Jahrhundert war also auch deshalb ein Jahrhundert Europas, weil die Anderen Maß an Europa nahmen." (Osterhammel 2009: 20). Distanzierungen zur grundsätzlichen Kritik an der Zentralstellung Europas auch bei Headley (2008), Huff (2010) und anderen. 
nicht nach gesetzlichen Vorschriften erlaubt ist, ist für die oben aufgeführten Weiterverwendungen des Materials die Einwilligung des jeweiligen Rechteinhabers einzuholen.

Weitere Details zur Lizenz entnehmen Sie bitte der Lizenzinformation auf http://creativecommons.org/ licenses/by/4.0/deed.de.

\section{Literatur}

Beck, Ulrich. 1983. Jenseits von Stand und Klasse? Soziale Ungleichheit, gesellschaftliche Individualisierungsprozesse und die Entstehung neuer sozialer Formationen und Identitäten. In Soziale Ungleichheiten, Hrsg. Reinhard Kreckel, 35-74. Göttingen: Schwartz.

Berger, Johannes. 2003. Neuerliche Anfragen an die Theorie der funktionalen Differenzierung. In Beobachter der Moderne: Beiträge zu Niklas Luhmanns „,Die Gesellschaft der Gesellschaft“, Hrsg. HansJoachim Giegel, Uwe Schimank, 207-230. Frankfurt a.M.: Suhrkamp.

Berger, Johannes. 2006. Die Einheit der Moderne. In Die Vielfalt und Einheit der Moderne: Kultur-und strukturvergleichende Analysen, Hrsg. Thomas Schwinn, 201-225. Wiesbaden: VS.

Berman, Harold J. 1991. Recht und Revolution: Die Bildung der westlichen Rechtstradition. Frankfurt a.M.: Suhrkamp.

Beutel, Albrecht. 2014. Elastische Identität: Die aufklärerische Aktualisierung reformatorischer Basisimpulse bei Johann Joachim Spalding. Zeitschrift für Theologie und Kirche 111:1-27.

Blumenberg, Hans. 1996. Die Legitimität der Neuzeit. Frankfurt a.M.: Suhrkamp.

Böckenförde, Ernst-Wolfgang. 2007. Die Entstehung des Staates als Vorgang der Säkularisation. In Der säkularisierte Staat: Sein Charakter, seine Rechtfertigung und seine Probleme im 21. Jahrhundert, 42-72. München: Carl Friedrich von Siemens Stiftung. [1976].

Brendle, Franz. 2011. Der Erzkanzler im Religionskrieg: Kurfürst Anselm Casimir von Mainz, die geistlichen Fürsten und das Reich 1629 bis 1647. Münster: Aschendorff.

Buschmann, Arno. 1994. Kaiser und Reich: Verfassungsgeschichte des Heiligen Römischen Reiches Deutscher Nation vom Beginn des 12. Jahrhunderts bis zum Jahre 1806 in Dokumenten. Teil 1. BadenBaden: Nomos.

Chakrabarty, Dipesh. 2000. Provincializing Europe: Postcolonial thought and historical difference. Princeton: Princeton University Press.

Conrad, Sebastian. 2013. Globalgeschichte: Eine Einführung. München: Beck.

Conrad, Sebastian, und Shalini Randeria. 2002. Jenseits des Eurozentrismus: Postkoloniale Perspektiven in den Geschichts- und Kulturwissenschaften. Frankfurt a.M.: Campus.

Descartes, René. 1972. Meditationen über die Grundlagen der Philosophie mit sämtlichen Einwänden und Erwiderungen. Hamburg: Meiner. ed. Artur Buchenau, [1641].

Dinges, Martin. 1991. Frühneuzeitliche Armenfürsorge als Sozialdisziplinierung? Probleme mit einem Konzept. Geschichte und Gesellschaft 17:5-29.

Dreier, Horst. 2002. Kanonistik und Konfessionalisierung: Marksteine auf dem Weg zum Staat. Juristenzeitung 57:1-13.

Durkheim, Emile. 1992. Über soziale Arbeitsteilung: Studie über die Organisation höherer Gesellschaften. Frankfurt a.M.: Suhrkamp. [1893].

Durkheim, Émile. 1970. Die Regeln der soziologischen Methode. Berlin, Neuwied: Luchterhand. [1895].

Eisenstadt, Shmuel N. 2000. Mutiple Modernities. In Multiple Modernities, Hrsg. Shmuel Eisenstadt, 1-29. London, New Brunswick: Transaction.

Epple, Angelika. 2012. Globalisierung/en. In Docupedia-Zeitgeschichte. http://docupedia.de/zg/epple_ globalisierung_v1_de_2012. Zugegriffen: 11. Juni 2012.

Erkens, Franz-Reiner. 2006. Herrschersakralität im Mittelalter: Von den Anfängen bis zum Investiturstreit. Stuttgart: Kohlhammer.

Gerhards, Jürgen. 1991. Funktionale Differenzierung und Prozesse der Entdifferenzierung. In Autopoiesis: Eine Theorie im Brennpunkt der Kritik, Hrsg. Hans R. Fischer, 263-280. Heidelberg: Carl-Auer.

Giddens, Anthony. 1996. Konsequenzen der Moderne. Frankfurt a.M.: Suhrkamp.

Greve, Jens, und Clemens Kroneberg. 2011. Herausforderungen einer handlungstheoretisch fundierten Differenzierungstheorie: Zur Einführung. In Soziale Differenzierung: Handlungstheoretische Zugänge in der Diskussion, Hrsg. Thomas Schwinn, Clemens Kroneberg, und Jens Greve, 7-23. Wiesbaden: VS.

Habermas, Jürgen. 1990a. Die Moderne - ein unvollendetes Projekt: Philosophisch-politische Aufsätze 1977-1990. Leipzig: Reclam. 
Habermas, Jürgen. 1990b. Strukturwandel der Öffentlichkeit: Untersuchungen zu einer Kategorie der bürgerlichen Gesellschaft. Frankfurt a.M.: Suhrkamp.

Hartmann, Wilfried. 2014. Gregor VII. und die Könige: Auf dem Weg zur Hierokratie? In Umstrittene Säkularisierung: Soziologische und historische Analysen zur Differenzierung von Religion und Politik, 2. Aufl., Hrsg. Karl Gabriel, Christel Gärtner, und Detlef Pollack, 101-133. Berlin: Berlin University Press.

Hattenhauer, Hans. 1970. Allgemeines Landrecht. Frankfurt a.M.: Metzner.

Hazard, Paul. 1939. Die Krise des europäischen Geistes. Hamburg: Hoffmann und Campe.

Headley, John M. 2008. The Europeanization of the world: on the origins of human rights and democracy. Princeton: Princeton University Press.

Heckel, Martin. 2007. Vom Religionskonflikt zur Ausgleichsordnung: Der Sonderweg des deutschen Staatskirchenrechts vom Augsburger Religionsfrieden 1555 bis zur Gegenwart. München: C. H. Beck.

Hölscher, Lucian. 1990. Die Religion des Bürgers: Bürgerliche Frömmigkeit und protestantische Kirche im 19. Jahrhundert. Historische Zeitschrift 250:595-630.

Hölscher, Lucian. 2005. Geschichte der protestantischen Frömmigkeit in Deutschland. München: C. H. Beck.

Huff, Toby E. 2010. Intellectual curiosity and the scientific revolution: a global perspective. Cambridge: Cambridge University Press.

Jansen, Nils. 2019. Recht und gesellschaftliche Differenzierung: Fünf Studien zur Genese des Rechts und seiner Wissenschaft. Tübingen: Mohr Siebeck.

Joas, Hans. 1992. Die Kreativität des Handelns. Frankfurt a.M.: Suhrkamp.

Joas, Hans. 2014. Gefährliche Prozessbegriffe: Eine Warnung vor der Rede von Differenzierung, Rationalisierung und Modernisierung. In Umstrittene Säkularisierung: Soziologische und historische Analysen zur Differenzierung von Religion und Politik, 2. Aufl., Hrsg. Karl Gabriel, Christel Gärtner, und Detlef Pollack, 603-622. Berlin: Berlin University Press.

Kant, Immanuel. 1900ff. Kritik der Urtheilskraft. In Gesammelte Schriften, Bd. 5, Hrsg. Preußische Akademie der Wissenschaften, 1900. Berlin: de Gruyter. [1790].

Kant, Immanuel. 1956. Kritik der reinen Vernunft. Nach der ersten und zweiten Original-Ausgabe. Hamburg: Meiner. [1787].

Kant, Immanuel. 1968. Die Religion innerhalb der Grenzen der bloßen Vernunft. In Gesammelte Schriften, Bd. 6, Hrsg. Preußische Akademie der Wissenschaften. Berlin:: de Gruyter. [1793].

Kaufmann, Franz-Xaver. 1989. Religion und Modernität: Sozialwissenschaftliche Perspektiven. Tübingen: Mohr Siebeck.

Kittsteiner, Heinz D. 1995. Die Entstehung des modernen Gewissens. Frankfurt a.M.: Suhrkamp.

Knöbl, Wolfgang. 2007. Die Kontingenz der Moderne: Wege in Europa, Asien und Amerika. Frankfurt a.M.: Campus.

Knöbl, Wolfgang. 2013. Aufstieg und Fall der Modernisierungstheorie und des säkularen Bildes ,moderner Gesellschaften“. In Moderne und Religion: Kontroversen um Modernität und Säkularisierung, Hrsg. Ulrich Willems, et al., 75-116. Bielefeld: transcript.

Koselleck, Reinhart. 1972ff. Einleitung. In Geschichtliche Grundbegriffe: Historisches Lexikon zur politisch-sozialen Sprache in Deutschland, Bd. 1, Hrsg. Otto Brunner, Werner Conze, und Reinhart Koselleck, XIII-XXIII. Stuttgart: Klett-Cotta.

Koselleck, Reinhart. 1973. Kritik und Krise: Eine Studie zur Pathogenese der bürgerlichen Welt. Frankfurt a.M.: Suhrkamp.

Koselleck, Reinhart. 1989. „Neuzeit“: Zur Semantik moderner Bewegungsbegriffe. In Vergangene Zukunft: Zur Semantik geschichtlicher Zeiten., 300-348. Frankfurt a.M.: Suhrkamp.

Krech, Volkhard. 2014. Über Sinn und Unsinn religionsgeschichtlicher Prozessbegriffe. In Umstrittene Säkularisierung: Soziologische und historische Analysen zur Differenzierung von Religion und Politik, 2. Aufl., Hrsg. Karl Gabriel, Christel Gärtner, und Detlef Pollack, 565-602. Berlin: Berlin University Press.

Kühne-Bertram, Gudrun. 1992. Das Schöne. In Historisches Wörterbuch der Philosophie, Bd. 8, Hrsg. Joachim Ritter, Karlsfried Gründer, 1370-1375. Basel, Stuttgart: Schwabe.

Leonhardt, Rochus. 2017. Religion und Politik im Christentum: Vergangenheit und Gegenwart eines spannungsreichen Verhältnisses. Baden-Baden: Nomos.

Lerner, Daniel. 1968. Modernization: social aspects. In International encyclopedia of the social sciences, Bd. 10, Hrsg. David L. Sills, 386-402. New York: MacMillan.

Lessing, Gotthold Ephraim. 1954. Nathan der Weise. In Gesammelte Werke, Bd. 2, 319-481. Berlin: Aufbau. [1778].

Lessing, Gotthold Ephraim. 1956. Die Erziehung des Menschengeschlechts. In Gesammelte Werke, Bd. 8, 590-615. Berlin: Aufbau. [1780]. 
Luhmann, Niklas. 1989. Die Ausdifferenzierung von Religion. In Gesellschaftsstruktur und Semantik, Bd. 3, 259-357. Frankfurt a.M.: Suhrkamp.

Luhmann, Niklas. 1997. Die Gesellschaft der Gesellschaft. Frankfurt a.M.: Suhrkamp.

Luther, Martin. 1520. Von der Freiheit eines Christenmenschen. WA, Bd. 7, 20-38.

Luther, Martin. 1523. Von weltlicher Obrigkeit. WA, Bd. 11, 246-280.

Luther, Martin. 1528. Vorrede zu Melanchthons „Unterricht der Visitatoren“

Maddison, Angus. 2007. Contours of the world economy, 1-2030 AD: Essays in macro-economic history. Oxford: Oxford University Press.

Marschalck, Peter. 1984. Die Bevölkerungsgeschichte Deutschlands im 19. und 20. Jahrhundert. Frankfurt a.M.: Suhrkamp.

Münch, Richard. 1991. Die Struktur der Moderne: Grundmuster und differentielle Gestaltung des institutionellen Aufbaus der modernen Gesellschaften. Frankfurt a.M.: Suhrkamp.

Nassehi, Armin. 2004. Die Theorie funktionaler Differenzierung im Horizont ihrer Kritik. Zeitschrift für Soziologie 33:98-118.

Nelson, Benjamin. 1974. Sciences and civilizations, „East“ and „West“. Boston Studies, Bd. XI, 445-493. German: Der Ursprung der Moderne: Vergleichende Studien zum Zivilisationsprozess. Frankfurt a.M.: Suhrkamp, 1986.

Nipperdey, Thomas. 1983. Deutsche Geschichte 1800-1866: Bürgerwelt und starker Staat. München: C. H. Beck.

Oexle, Otto Gerhard. 1978. Die funktionale Dreiteilung der „Gesellschaft“ bei Adalbero von Laon: Deutungsschemata der sozialen Wirklichkeit im frühen Mittelalter. Frühmittelalterliche Studien 12:1-54.

Offe, Claus. 1989. Fessel und Bremse: Moralische und institutionelle Aspekte intelligenter Selbstbeschränkung. In Zwischenbetrachtung: Im Prozess der Aufklärung, Hrsg. Axel Honneth, et al., 739-774. Frankfurt a.M.: Suhrkamp.

Osterhammel, Jürgen. 2009. Die Verwandlung der Welt: Eine Geschichte des 19. Jahrhunderts. München: C. H. Beck.

Osterhammel, Jürgen. 2016. Hierarchien und Verknüpfungen: Aspekte einer globalen Sozialgeschichte. In Geschichte der Welt: 1750-1870: Wege zur modernen Welt, Hrsg. Akira Iriye, et al., 627-836. München: C. H. Beck.

Otto, Rüdiger. 2016. Religion und Stadt: Kirchengeschichte Leipzigs von 1650 bis 1815. Beucha; Markkleeberg: Sax.

Pomeranz, Kenneth. 2000. The great divergence: China, Europe, and the making of the modern world economy. Princeton: Princeton University Press.

Reuter, Astrid. 2009. Charting the boundaries of the religious field: Legal conflicts over religion as struggles over blurring borders. Journal of Religion in Europe 2:1-20.

Reuter, Astrid. 2014. Religion in der verrechtlichten Gesellschaft: Rechtskonflikte und öffentliche Kontroversen um Religion als Grenzarbeiten am religiösen Feld. Göttingen: Vandenhoeck \& Ruprecht.

Riches, Theo. 2012. Episcopal actors and royal action: Distinguishing religion and politics in the eleventh century. Präsentation Februar 14th 2012 DFG, Bonn

Schiller, Friedrich. 1993. Über die ästhetische Erziehung des Menschen in einer Reihe von Briefen. In Sämtliche Werke, Bd. 5, Hrsg. Gerhard Fricke, Herbert G. Göpfert, 570-669. München, Wien: Hanser. [1795].

Schilling, Heinz. 1988. Aufbruch und Krise: Deutschland 1517-1648. Berlin: Siedler.

Schilling, Heinz. 2009. Das konfessionelle Europa. In Europäische Religionsgeschichte: Ein mehrfacher Pluralismus, Hrsg. Hans G. Kippenberg, Jörg Rüpke, und Kocku von Stuckrad, 289-338. Göttingen: Vandenhoeck \& Ruprecht.

Schimank, Uwe. 1988. Gesellschaftliche Teilsysteme als Akteurfiktionen. Kölner Zeitschrift für Soziologie und Sozialpsychologie 40:619-639.

Schimank, Uwe. 2005. Differenzierung und Integration der modernen Gesellschaft: Beiträge zur akteurzentrierten Differenzierungstheorie. Wiesbaden: VS.

Schimank, Uwe. 2011. Gesellschaftliche Differenzierungsdynamiken - ein Fünf-Fronten-Kampf. In Soziale Differenzierung: Handlungstheoretische Zugänge in der Diskussion, Hrsg. Thomas Schwinn, Clemens Kroneberg, und Jens Greve, 261-284. Wiesbaden: VS.

Schindling, Anton. 1997. Konfessionalisierung und Grenzen der Konfessionalisierbarkeit. In Die Territorien des Reichs im Zeitalter der Reformation und Konfessionalisierung: Land und Konfession 1500-1650, Bd. 7, Hrsg. Anton Schindling, Walter Ziegler, 9-44. Münster: Aschendorff.

Schlaich, Klaus. 1969. Kollegialtheorie: Kirche, Recht und Staat in der Aufklärung. München: Claudius.

Schleiermacher, Friedrich. 1984. Der christliche Glaube. Bd. 1. Berlin: de Gruyter. ed. Martin Redeker, [1830/31]. 
Schleiermacher, Friedrich. 1999. Über die Religion: Reden an die Gebildeten unter ihren Verächtern. Berlin: de Gruyter. ed. Günter Meckenstock, [1799].

Schlögl, Rudolf. 2013. Alter Glaube und moderne Welt: Europäisches Christentum im Umbruch 1750-1850. Frankfurt a.M.: Fischer.

Schneider, Wolfgang Ludwig. 2011. Religion und funktionale Differenzierung. In Soziale Differenzierung: Handlungstheoretische Zugänge in der Diskussion, Hrsg. Thomas Schwinn, Clemens Kroneberg, und Jens Greve, 181-210. Wiesbaden: VS.

Schwinn, Thomas. 2001. Differenzierung ohne Gesellschaft: Umstellung eines soziologischen Konzepts. Weilerswist: Velbrück Wissenschaft.

Schwinn, Thomas. 2006. Die Vielfalt und die Einheit der Moderne: Perspektiven und Probleme eines Forschungsprogramms. In Die Vielfalt und Einheit der Moderne: Kultur- und strukturvergleichende Analysen, Hrsg. Thomas Schwinn, 7-34. Wiesbaden: VS.

Schwinn, Thomas. 2013. Zur Neubestimmung des Verhältnisses von Religion und Moderne: Säkularisierung, Differenzierung und multiple Modernitäten. Kölner Zeitschrift für Soziologie und Sozialpsychologie 65(S1):73-97.

Sehling, Emil. 1914. Geschichte der protestantischen Kirchenverfassung, 2. Aufl., Leipzig: B.G. Teubner.

Earl of Shaftesbury, A.A.C. 1981. The moralists: A philosophical rhapsody. In Moral and political philosophy, Bd. II/1, Hrsg. W. Benda, G. Hemmerich, und K. Schödlbauer. Stuttgart: Frommann-Holzboog.

Siebenhüner, Kim. 2007. Glaubenswechsel in der Frühen Neuzeit: Chancen und Tendenzen einer historischen Konversionsforschung. Zeitschrift für Historische Forschung 34:243-272.

Southern, Richard W. 2001. Scholastic humanism and the unification of Europe. Oxford: Wiley-Blackwell. 2 volumes (1: Foundations, 2: The heroic age).

Spalding, Johann Joachim. 2002. Ueber die Nutzbarkeit des Predigtamtes und deren Beförderung. In Kritische Ausgabe, Bd. I/3, Hrsg. Tobias Jersak. Tübingen: Mohr Siebeck. [1772].

Spalding, Johann Joachim. 2006. Die Bestimmung des Menschen. In Kritische Ausgabe, Bd. I/1, Hrsg. Albrecht Beutel, Daniela Kirschkowski, und Dennis Prause. Tübingen: Mohr Siebeck. [1748].

Spencer, Herbert. 1998. The principles of sociology. London: Williams and Norgate. 3 volumes.

Stegmann, Andreas. 2014. Luthers Auffassung vom christlichen Leben. Tübingen: Mohr Siebeck.

Stollberg-Rilinger, Barbara. 2006. Europa im Jahrhundert der Aufklärung. Stuttgart: Reclam.

Stollberg-Rilinger, Barbara. 2019. Teufels Werk und Gottes Beitrag? Katholische Konfessionalisierung und Katholische Aufklärung als Faktoren der Modernisierung

Stollberg-Rilinger, Barbara, und Andreas Pietsch. 2013. Konfessionelle Ambiguität: Uneindeutigkeit und Verstellung als religiöse Praxis in der Frühen Neuzeit. Gütersloh: Gütersloher Verlagshaus.

Strayer, Joseph R. 1970. On the medieval origins of the modern state. Princeton: Princeton University Press.

Strohm, Christoph. 2008. Calvin und die religiöse Toleranz. In 1509 - Johannes Calvin - 2009: Sein Wirken in Kirche und Gesellschaft: Essays zum 500. Geburtstag, Hrsg. Martin Ernst Hirzel, Martin Sallmann, 219-236. Zürich: TVZ.

Tellenbach, Gerd. 1988. Die westliche Kirche vom 10. bis zum frühen 12. Jahrhundert. Göttingen: Vandenhoeck \& Ruprecht.

Troeltsch, Ernst. 1911. Die Bedeutung des Protestantismus für die Entstehung der modernen Welt. München. Berlin: Oldenbourg.

Tyrell, Hartmann. 2008. Soziale und gesellschaftliche Differenzierung: Aufsätze zur soziologischen Theorie. Wiesbaden: VS.

Tyrell, Hartmann. 2010. Katholische Weltkirche und Religionsfreiheit: Christentumsgeschichtliche und differenzierungstheoretische Überlegungen. In Religionsfreiheit und Pluralismus: Entwicklungslinien eines katholischen Lernprozesses, Hrsg. Karl Gabriel, Christian Spieß, und Katja Winkler, 197-260. Paderborn: Schöningh.

Wallmann, Johannes. 2012. Kirchengeschichte Deutschlands seit der Reformation, 7. Aufl., Tübingen: Mohr Siebeck.

Walther, Manfred. 1971. Metaphysik als Anti-Theologie: Die Philosophie Spinozas im Zusammenhang der religionsphilosophischen Problematik. Hamburg: Meiner.

Wassilowsky, Günther. 2016. Einleitung. In Das Konzil von Trient und die katholische Konfessionskultur (1563-2013), Hrsg. Peter Walter, Günther Wassilowsky, 1-13. Münster: Aschendorff.

Weber, Max. 1920. Vorbemerkung. In Gesammelte Aufsätze zur Religionssoziologie I, Hrsg. Max Weber, 1-16. Tübingen: Mohr Siebeck. [1988].

Weinfurter, Stefan. 2006. Canossa: Die Entzauberung der Welt. München: Beck.

Wrigley, Edward A. 1969. Bevölkerungsstruktur im Wandel: Methoden und Ergebnisse der Demographie. München: Kindler. 